\title{
SPARCO: Stochastic Performance Analysis with Reliability and Cooperation for Underwater Wireless Sensor Networks
}

\author{
Sheeraz Ahmed, ${ }^{1,2}$ Nadeem Javaid, ${ }^{1}$ Ashfaq Ahmad, ${ }^{1}$ Imran Ahmed, ${ }^{2}$ Mehr Yahya Durrani, \\ Armughan Ali, ${ }^{3}$ Syed Bilal Haider, ${ }^{3}$ and Manzoor Ilahi ${ }^{1}$ \\ ${ }^{1}$ COMSATS Institute of Information Technology, Islamabad, Pakistan \\ ${ }^{2}$ Institute of Management Sciences (IMS), Peshawar, Pakistan \\ ${ }^{3}$ COMSATS Institute of Information Technology, Attock, Pakistan \\ Correspondence should be addressed to Nadeem Javaid; nadeemjavaidqau@gmail.com
}

Received 22 November 2015; Accepted 5 January 2016

Academic Editor: Wei Cao

Copyright (C) 2016 Sheeraz Ahmed et al. This is an open access article distributed under the Creative Commons Attribution License, which permits unrestricted use, distribution, and reproduction in any medium, provided the original work is properly cited.

\begin{abstract}
Reliability is a key factor for application-oriented Underwater Sensor Networks (UWSNs) which are utilized for gaining certain objectives and a demand always exists for efficient data routing mechanisms. Cooperative routing is a promising technique which utilizes the broadcast feature of wireless medium and forwards data with cooperation using sensor nodes as relays. Here, we present a cooperation-based routing protocol for underwater networks to enhance their performance called Stochastic Performance Analysis with Reliability and Cooperation (SPARCO). Cooperative communication is explored in order to design an energy-efficient routing scheme for UWSNs. Each node of the network is assumed to be consisting of a single omnidirectional antenna and multiple nodes cooperatively forward their transmissions taking advantage of spatial diversity to reduce energy consumption. Both multihop and single-hop schemes are exploited which contribute to lowering of path-losses present in the channels connecting nodes and forwarding of data. Simulations demonstrate that SPARCO protocol functions better regarding end-to-end delay, network lifetime, and energy consumption comparative to noncooperative routing protocol-improved Adaptive Mobility of Courier nodes in Threshold-optimized Depth-based routing (iAMCTD). The performance is also compared with three cooperation-based routing protocols for UWSN: Cognitive Cooperation (Cog-Coop), Cooperative Depth-Based Routing (CoDBR), and Cooperative Partner Node Selection Criteria for Cooperative Routing (Coop Re and dth).
\end{abstract}

\section{Introduction}

UWSNs consist of sensors and vehicles that are deployed over a given region to perform collaborative monitoring tasks. These networks offer variety of applications like tactical surveillance, environmental monitoring, assisted navigation, resource investigation, and disaster prevention. The physical layer has a strong influence on UWSNs due to the presence of acoustic waves. Acoustic waves are the most accurate source of reaching up to desirable range and rate of data transmission in UW communications. Radio waves do not support required data rate and range as they get absorbed in water very quickly. New achievements in underwater acoustic (UWA) communications, however, make adequate data forwarding over long distances. Many techniques have been investigated on developing networking solutions for UWSNs including acoustic channel modeling, physical layer transmission analysis, and networking protocols.

Acoustic waves experience large delay spreads as they move at the speed of $1500 \mathrm{~m} / \mathrm{s}$. This speed makes one-fifth speed as that of radio waves. Path-loss, in the case of radio channels, depends solely on the length of the link; but the acoustic waves in UW experience both link length and frequency dependent path-losses. Bubbles and suspended particles in water make wide dispersion in acoustic waves. Also, the reflections from surface of water as well as from the bed of the sea increase the channel fading. All these factors 
have to be taken into account for the design of UWA wireless systems.

Having now sufficient technological advancements made in the field of radio communications, researches are trying to enhance the working of UW systems using modern techniques adopted from radio communications. A promising technique is cooperative communication, already being used in terrestrial WSNs. It is a potential approach for distributed UWSNs to upgrade the quality of link connecting sensors as well as the reliability in both point-to-point and multipoint environments, having multiple relays doing cooperation. Wireless network designs take into account the diversity to improve the overall successful transmissions by allowing duplicate signals at the receiver. In contrast to this approach, Multiple-Input Multiple-Output (MIMO) technique also uses a promising mechanism to improve Signal-to-Noise Ratio (SNR) by enhancing diversity gain. But, the technique needs extra equipment cost for each sensor with much complexity. A different approach for gaining diversity is to utilize several sensors cooperating with each other to upgrade the quality of communication channel. In variation to an individual sensor having an antenna array, duplicate data is forwarded by an array of distributed antennas comprised of several sensors to reach the end-point but introducing some delay. Spatial diversity concept has directed regular efforts to its use in wireless networks particularly in WSNs. If various paths existing between two end devices are not dependent on each other and have adequate working, channel efficiency can be improved by forwarding multiple duplicates of data along these links and merging them at the destination. The total error probability decreases since the paths are independent, which makes the channel and system performance increase. However, in general case of WSNs, nodes are very small to that needed for the support of such distributed antennas. Hence, to combat such issues, the idea of cooperative routing is proposed. Cooperation is defined as a group of entities working together to achieve a common goal while sharing each other's resources. In these systems, transmitter forwards one copy of data packets to a node acting as relay. The relay then decodes or amplifies each data packet as the scheme suggests and reforwards it to the final receiver. Relay node uses a path which is generally different from the direct path. The destination merges or utilizes both of the received signals to extract the forwarded information.

Cooperative diversity, an alternative to combat fading in wireless channels, allows distributed users to help relay information of each other to explore inherent spatial diversity present in channels. Various cooperation-based protocols have been proposed in literature like fixed relaying protocol, adaptive relaying protocol, user cooperation protocol, and coded cooperation schemes. In fixed relaying schemes, such as Amplify-and-Forward (AF) and Decodeand-Forward (DF), relays provide assistance to provide the source information. In AF scheme, the relays amplify and forward the information, whereas, in DF scheme, the relays decode the received information and then transmit it to the receiver. Although, in DF, the relay forwards the decoded information to the receiver, however, this scheme has adequate degradation in performance if the relay does not decode the transmitters information properly.

\section{Related Work}

Earlier efforts to investigate the underwater behavior were just depending on the technology available for terrestrial sensor networks. However, UWSNs show several structural design differences in comparison to terrestrial networks, especially due to the water used as the transmission medium and the signal required for data transmission. Design of a suitable structure for UWSNs is also complex due to the behavior of communication system.

An efficient scheme for UWSN, Coop (Re and dth) [1], employs cooperative routing which involves data transmission via partner node/relay towards sink. In this paper, two different partner node selection criteria are implemented and compared. The authors have considered source node depth-threshold (dth), potential relays depth, and residual energy $(\mathrm{Re})$ as one criterion and SNR of the link connecting source node with relay or destination as another criterion for selection parameters. Cog-Coop [2] is an efficient scheme for maximization of network lifetime using residual energy of the nodes. It improves the spectrum sensing performance along with having better energy consumption of the sensors. Optimal conditions are attained based on the standard optimization methods, to find the priority of sensors for spectrum sensing. They showed that cooperation among cognitive sensors is necessary for lowering the fading as well as shadowing effects and, hence, correct sensing. Cooperative Depth-Based Routing (CoDBR) for UWSNs [3] is a cooperation-based routing protocol proposed to enhance network performance. Efficient nodes for relaying are selected on the basis of information of depth. Source node transmits data to the sink node by relay sensors using cooperation. In [4], authors propose forwarding function based routing scheme iAMCTD for UWSNs. The reactive protocol enhances the underwater network lifetime by the best possible mobility design of sink.

A communication path based routing scheme called Relative Distance Based Forwarding (RDBF) is presented in [5]. The authors have used a mathematical factor to judge and measure the level of suitability for a sensor to relay the data packets. Few nodes are involved in forwarding mechanism, helping in reduction of end-to-end delay as well as energy consumption. The protocol also controls the forwarding time of the multiple transmitters to minimize the duplicate data transmission. In [6], the authors have presented routing protocols for cylindrical networks and developed mathematical models to achieve best selection of path for information forwarding. Their results showed that the suggested 4-chain based protocol performs efficiently in terms of lifetime of the network, path-loss, end-to-end delay, and packet sending rate.

The problem of tracking UW moving targets is tackled in [7]. For 3-dimensional UW maneuvering target tracking, the cooperating model technique is added with the particle filter to handle with qualms. Simulation outcomes explain that the suggested scheme is a good alternate for customary sensor-based or imaging-based approaches. 
In [8], the authors propose cooperation-based scheme based on both Quality-of-Service (QoS) and energy consumption. To amalgamate the two strictures in the scheme, a competitive approach is adopted at each sensor by utilizing Multiagent Reinforcement-Learning (MRL) algorithm. The suggested scheme guarantees improved working regarding packet loss rate and end-to-end delay, while considering the energy consumption of the network. The main idea of cooperative communication is to utilize the resources of more than one node to transmit data. Hence, by resource sharing between sensors, quality of transmission is upgraded. ACOAAFSA forwarding protocol is presented in [9] which has the positive characteristics of Artificial Fish Swarm Algorithm (AFSA) and Ant Colony Optimization Algorithm (ACOA). The fusion algorithm reduces the energy consumption and transmission delay of the existing routing protocols and promises to improve the robustness. The Remotely Powered UWA Sensor Networks (RPUASN) protocol proposed in [10] shows that sensors harvest and store the power given by an external acoustic source, upgrading their stability period to a large extent. The desired number of sensors and the region which is trusted to be sensed by the sensors are examined regarding range, power, directivity, and frequency of the external source.

Authors in [11] investigate a 3-dimensional underwater network which aims to cover up the coverage hole problem and reduces the energy consumption of the sensors along with enhancing the collection of data. They used an appleslice technique to build multiple sectors to enclose the hole and to guarantee the continuity of routing path. Results show the working upgradation of successful packet delivery ratio and reduction of message overhead and power consumption. In [12], the authors propose a time-based priority forwarding method to prevent flooding. Result outcomes indicate that the routing protocol attains outstanding working regarding packet delivery ratio, energy consumption, and end-to-end delay.

In [13], authors have presented a detailed measurement of fading and path-loss characteristics for sensors in both flat and irregular outdoor land using mathematical path-loss model. Research in [14] addresses the diverse challenges faced in an underwater environment and the advancements being in progress. According to authors, due to the cost of sea tests and the lack of standards, there are no operational UW networks but only experimental demonstrations. Efficient and scalable protocols are needed if bigger deployments are to be expected. Authors in [15] have focused on a survey of existing routing protocols in UWSNs. Firstly, they have classified routing protocols into two categories based on a route decision maker. Then the performance of existing routing protocols is compared. Furthermore, future research issues of routing protocols in UWSNs are carefully analyzed.

An approach to mapping of a wireless connected UW Robotic Fish (URF) is presented in [16]. It is based on both Cooperative Localization Particle Filter (CLPF) scheme and Occupancy Grid Mapping Algorithm (OGMA). CLPF shows that no prior information about the kinematic model of URF is needed to attain correct 3D localization. Simulations show the effectiveness of the suggested scheme. In [17], researchers present a contention-free multichannel Media Access Control (MAC) scheme for underwater networks that performs well even when sensors go through uneven and heavy traffic conditions. Simulations show that the scheme saves energy and is quite reasonable for a bursty-loaded environment. In [18], the authors describe a new acoustic modem ITACA for UWSN, which includes a low-power asynchronous wake-up system implementation. This modem for UWA forwarding is based on a low-cost radio frequency integrated circuit. The characteristic enables a much less power dissipation of $10 \mathrm{~W}$ in stand-by mode. In [19], the authors introduce an UW sensor node equipped with an embedded camera. Utilizing this platform, the authors present a speedy and much correct debris detection algorithm, based on compressive sensing theory to consider the challenges of UWA environments. Experimental results identify that their approach is trustworthy and suitable for debris detection using camera sensors in UW environments.

In [20], the authors present localization schemes using color filtering technology called Projection-Color Filtering Localization (PCFL) and Anchor-Color Filtering Localization (ACFL). Both algorithms focus at jointly accomplishing correct localization of UWA sensors with much less energy consumption. They both accept the overlapping signal area of task anchors which communicate with the mobile node directly as the current sampling region. Comparison of the nearness degrees of the RGB sequences between samples and the mobile node filters out the samples. Simulations indicate that the suggested techniques have quite better localization performance and can timely localize the mobile node. In [21], the authors have presented a scheme Ultrasonic Frog Calling Algorithm (UFCA) for UWSNs that targets achieving energy-efficient routing under harsh UW conditions. In this scheme, the selection of forwarding nodes is adopted in the same way of calling behavior of frogs for mating. The sensor nodes located in worse places go into sleep mode for energy conservation. In [22], the authors proposed a balanced transmission mechanism for decreasing energy consumption. They divided the data transmission phase into two phases and then determined single-hop or multihop data transmission of the node to the sink depending on the residual energy of the node. The authors in [23] presented a cooperationbased Harvest-Then-Cooperate (HTC) scheme for WSNs in which the source and relay harvest energy from the access point in the downlink and work cooperatively in the uplink for the source information transmission. The impacts of the system parameters, like relay number, time allocation, and relay position, on the throughput performance were investigated. Modified Double-Threshold Energy Detection (MDTED) scheme is presented in [24] which is a cooperative spectrum sensing scheme for WSNs. The paper incorporates location and channel to improve the clustering mechanism and hence collaborative sensing ability.

\section{Motivation}

Majority of network applications consist of battery-powered nodes having limited transmission-reception range. Thus, cooperative communication or alternately cooperative 
routing is particularly needed for such networks, in which sensor nodes share their resources among each other. Migrating from lengthy but weak links to shorter as well as strengthened links can minimize the load on the path connecting sensors. Different available paths present between sensors and sinks provide means for the new design options regarding scheduling and routing.

Cog-Coop uses both spectrum sensing information and residual energy of sensors to select the optimal forwarder nodes. It improves the spectrum sensing performance along with having better energy consumption of the sensors. Optimal conditions are attained centered on the typical optimization approaches to find the priority of sensors for spectrum sensing. They showed that cooperation among cognitive nodes is necessary for lowering the fading as well as shadowing effects and, hence, correct sensing. Also, the forwarding node selection criteria are suggested to save energy and deal with the issue of direct communications with the fusion center. It is a cooperative routing protocol and redundant transmissions consume a lot of energy. Therefore, packets are forwarded over a single lossy channel in a multihop order. Due to noise and multipath fading in UW environment, signal suffers high bit error rate. In iAMCTD, a routing scheme is proposed to maximize the lifetime of reactive UWSNs. iAMCTD considers signal quality as well as residual energy for routing metrics. It is a prototype scheme in localization-free and flooding centered data forwarding for UW scenarios. It improves the network throughput and minimizes packet drop ratio to a larger extent by using formulated forwarding functions. iAMCTD faces redundant transmissions resulting in major energy consumption.

Coop ( Re and dth) aims to solve the issues of EEDBR and iAMCTD via cooperative diversity. This protocol involves data transmission through the use of partner nodes/relays that use cooperation to route the data to the sink. This improves the rate of fruitful packets delivery to the sink as in the chances of link disaster; at least one link is present for forwarding of packets reliably to the final end. The scheme considers a node link state information along with its residual energy and depth as selection criteria. So, Coop (Re and dth) is consuming more broadcasting energy than AMCTD and EEDBR. This presents us with the trade-off between reliability and energy conservation. Also, the protocol does not consider any transmission impairments present in the underwater environment. CoDBR aims to solve the issues of EEDBR via cooperative diversity. The protocol chooses the forwarder sensor using two relays selected on the basis of least depth that route their packets to sink, cooperatively. This enhances the successful data delivery rate because if a link fails, then at least another path is present for forwarding packets successfully to the sink. As CoDBR considers one source node and two relay sensors to forward packets to next hop, hence CoDBR consumes three times greater broadcasting energy than EEDBR. It is also a trade-off between link availability and energy conservation. In order to address the issues of all these four protocols, we have tried to propose a new protocol by the name of SPARCO.

In SPARCO scheme, we suggest an approach to forward information through UWSNs with much lower path-loss over a link using the features of single-hop and multihop. The protocol uses a cost function to find the most suitable path to sink. This function is computed using node distances from the destination and their residual energies. Simulations depict that SPARCO scheme enhances the network stability period with much reduced path-loss, to a large extent.

The research takes into account an underwater acoustic environment, where the channel is largely attenuated by fading and other noise effects. The sensed signal by the sensors is modeled by a Rayleigh random variable. The proposed mechanism leads to enhancing the reliability of the underwater channel by the use of cooperative routing. In this work, we consider the technique of FRC for signal combining. Cooperative diversity is obtained without using various antennas. This is particularly useful when frequency, time, and diversity by the use of several antennas are not appropriate. This motivated us not only to the utility of cooperation in underwater environment, but also to evaluate its impact on system performance.

\section{SPARCO: System Model}

Figure 1 shows a 5-node UWSN model, where $S$ and $D$ are the source and destination nodes, respectively. Data transferred by $S$ is received by nodes $R 1, R 2$, and $R 3$. Having done with the initial transmission, nodes $S, R 1$, and $R 2$ have the data and cooperatively transmit the data to $R 3$ and then $D$. Let the minimum energy path from $S$ to $D$ is determined through $R 2$; that is, $S \rightarrow R 2 \rightarrow D$. Node $R 1$, present within the transmission range of $S$ to $R 2$, receives the data forwarded from $S$. The dark lines in Figure 1 show the direct communication paths whereas the dotted lines indicate the cooperative routes in case the direct transfer path is not available or not feasible to use.

It is assumed that each sensor dynamically adjusts its transmission power to manage its range and further that various sensors cooperating in forwarding the data to a single receiving node can delay their transmission for perfect phase synchronization at $D$.

It is also assumed that the information is routed from $S$ to $D$ in transmission slots one after another. For each slot, a sensor is selected to broadcast the data to a group of sensors that received the information. This will help the nodes to cooperate for transmitting the data to another group of sensors.

The routing problem is tackled as a multistage decision problem. The decision is made at each stage to select the receiving as well as forwarding group of sensors and the transmission power level among all sensors of that stage as well. The purpose is to get the information to the final point with minimum energy.

Let the transmitting set at any stage $k$ be denoted by $S_{n}$ and let the receiving set be denoted by $R_{m}$. The link cost between $S_{n}$ and $R_{m}$, denoted by $C\left(S_{n}, R_{m}\right)$, is the minimum power needed for data transmission from $S_{n}$ to $R_{m}$. Here, $S_{n}=\left\{s_{1}, s_{2}, \ldots, s_{n}\right\}$ and $R_{m}=\left\{r_{1}, r_{2}, \ldots, r_{m}\right\}$.

To derive the equations for the costs on the link connecting nodes, we consider 4 different possibilities [24]. 


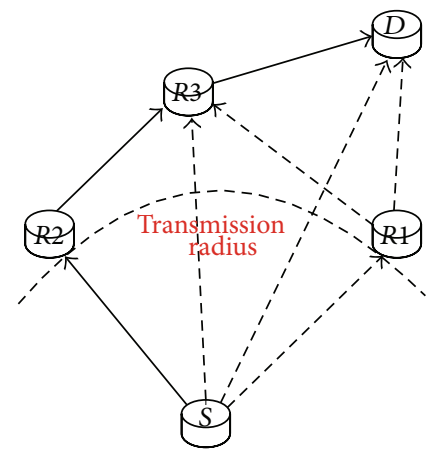

FIGURE 1: Multihop routing.

(i) $\{$ Point-to-Point Link\}, That Is, $(n=1, m=1)$. A single source node is transmitting to a single receiving node within a time-slot.

(ii) $\{$ Point-to-Multipoint/Broadcast Link\}, That Is, $(n=1, m>$ 1). One node is transmitting to various receiving nodes.

(iii) $\{$ Multipoint-to-Point/Cooperative Link\}, That Is, $(n>$ $1, m=1)$. Various nodes are cooperating in forwarding the same data to one receiver. The signal is aggregated at the receiver and full decoding is only possible if the received SNR is more than the threshold $\mathrm{SNR}_{\min }$.

(iv) $\{$ Multipoint-to-Multipoint Link\}, That $I s,(n>1, m>$ 1). Several nodes transmitting data to various other nodes makes redundant transmission at various receivers which is not feasible. Hence it is dealt with as a MIMO case.

The preceding four cases are discussed individually with their link-cost formulations.

Case 1 (\{point-to-point link\}, i.e., $(n=1, m=1)$ ). Here $S_{n}=$ $\left\{s_{1}\right\}$ and $R_{m}=\left\{r_{1}\right\}$. Let the wireless channel in underwater between these nodes be described by 2 factors: magnitude or attenuation factor $\alpha_{n m}$ and phase delay $\phi_{n m}$ as shown in Figure 2. The generated signal is managed by a scaling factor $f$. In UW applications, $f$ is a complex factor adopting both phase and power adjustment by the sender. As there is a single receiver in this case, hence the phase can be ignored.

The received signal is computed as follows [24]:

$$
x(t)=\alpha e^{j \phi} f \widehat{p}(t)+N(t),
$$

where $\widehat{p}(t)$ is the unit-power generated signal and $N(t)$ is the cumulative receiver noise in UW having power $P_{N}$. Net generated power is $P_{T}=f^{2}$ and SNR at the receiving node is $\alpha^{2}\left|f^{2}\right| / P_{N}$. SNR needs to be more than the threshold value $\mathrm{SNR}_{\min }$. Minimum power needed is $\bar{P}_{T}$ and hence the pointto-point $C_{p-p}\left(s_{1}, r_{1}\right)$ is given by [24]

$$
\begin{aligned}
C_{p-p}\left(s_{1}, r_{1}\right) & =\bar{P}_{T}=\frac{\mathrm{SNR}_{\min } P_{N}}{\alpha^{2}}, \\
\bar{P}_{T} & \propto \frac{1}{\alpha^{2}}=\frac{1}{d^{2}}
\end{aligned}
$$

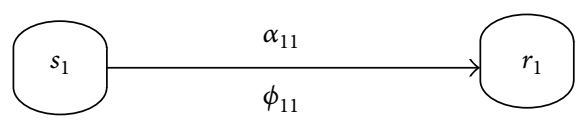

FIGURE 2: Single-input-single-output linkage.

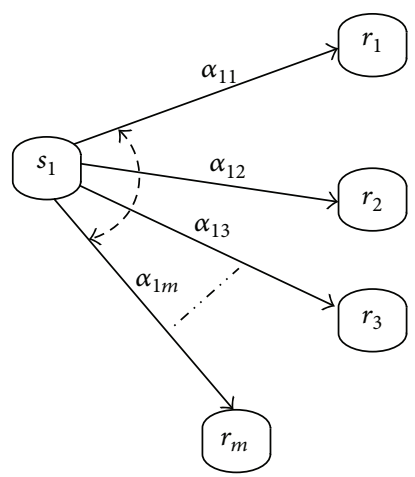

FIgURE 3: Broadcast linkage.

that is, $C_{p-p}$ is inversely proportional to square of the distance $d$ between $s_{1}$ and $r_{1}$.

Case 2 (\{point-to-multipoint link\}, i.e., $(n=1, m>1))$. In this case, $S_{n}=\left\{s_{1}\right\}$, and $R_{m}=\left\{r_{1}, r_{2}, \ldots, r_{m}\right\}$ as shown in Figure 3; thus $m$ similar SNR limitations should be satisfied at receiver nodes. The signal transmitted by $s_{1}$ is received by all those sensors that are within the transmission radius and proportional to the transmission power $P_{T}$. Minimum power needed for this broadcast transmission is represented by $C_{p-m}\left(s_{1}, R_{m}\right)$, expressed as

$$
\begin{aligned}
& C_{p-m}\left(s_{1}, R_{m}\right)=\operatorname{maximize}\left\{C_{11}\left(s_{1}, r_{1}\right), C_{12}\left(s_{1}, r_{2}\right), \ldots,\right. \\
& \left.C_{1 m}\left(s_{1}, r_{m}\right)\right\} .
\end{aligned}
$$

As the receiver nodes can be in various dimensions in terms of placement, so we use Principal Component Analysis (PCA) technique for this maximization problem. PCA is a reknowned technique for dimension reduction and exploratory data analysis [25].

For a group of sensed $v$-dimensional data vectors $\left\{v_{t}\right\}$, $t \in\{1,2, \ldots, T\}$, the $q$ principal axes $w_{j}, j \in\{1,2, \ldots, q\}$, are those orthonormal axes onto which the retained variance under projection is maximal. The vectors $w_{j}$ are expressed by the $q$ dominant eigenvectors (i.e., ones having maximum associated eigenvalues $\lambda_{j}$ ) of the sample covariance matrix, $M=E\left[(v-\mu)(v-\mu)^{T}\right]$, so that $M_{w_{j}}=\lambda_{j} \cdot w_{j}$. The $q$ principal components of the observed vector $v_{t}$ are expressed in terms of the vector $x_{t}=W^{T}\left(v_{t}-\mu\right)$, where $W^{T}=\left(w_{1}, w_{2}, \ldots, w_{q}\right)^{T}$. The variables $x_{j}$ are such decorellated that the covariance matrix $E\left[x \cdot x^{T}\right]$ is diagonal with elements $\lambda_{j}$.

Case 3 (\{multipoint-to-point link\}, i.e., $(n>1, m=1))$. In this case, $S_{n}=\left\{s_{1}, s_{2}, \ldots, s_{n}\right\}$, and $R_{m}=\left\{r_{1}\right\}$ as shown in Figure 4. Here $n$ transmitting nodes adjust their phases such that the signal is received at the receiver in phase. 


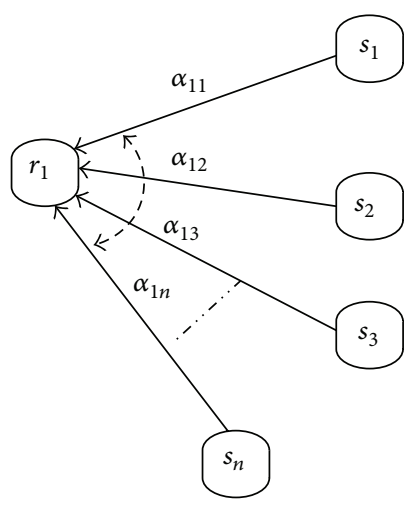

FIGURE 4: Cooperative linkage.

As we are considering PCA in this modeling, so a latent variable model is required to relate the group of $v$ dimensional observed data vectors $\left\{v_{t}\right\}$ to a corresponding group of $q$-dimensional latent variables $\left\{x_{m}\right\}$ such that [24]

$$
l=y(x ; \theta)+\epsilon,
$$

where $y(x ; \theta)$ is a function of the latent variable $x$ with parameter $\theta$ and $\epsilon$ is an $x$-independent noise process. Equation (4) is quite similar to (1) which is utilized for a single point-to-point node data transfer.

Generally $q<v$ so that the latent variable offers a more promising description of the data and, in Case 3, $q=1$; thus only a single receiving node is being used here. In standard form, the mapping $(x ; \theta)$ is linear, so

$$
l=W x+\mu+\epsilon,
$$

where the latent variables $x \sim \mathcal{N}(0, I)$ have a unit Gaussian distribution. The noise model is also Gaussian and $\epsilon \sim$ $\mathcal{N}(0, \psi)$ having $\psi$ diagonal, the $(v \times q)$ parameter matrix in our case, that is, $(v \times 1)$. Hence, the model for $l$ is also normal, that is, $\mathcal{N}(\mu, C)$, where the covariance $C=\psi+W W^{T}$.

The model is selected because of the diagonality of $\psi$ and the sensed variables $l$ are independent of latent variables, $x$. In this case, the latent variables are the parameters $\alpha_{n 1}$ and $\phi_{n 1}$. Analytic solution for $W$ and $\psi$ does not exist in literature and their values are to be computed by iterative methods.

For the model of (5), with an isotropic noise, $\psi=\sigma^{2}$. The noise model $\epsilon \sim \mathcal{N}\left(0, \sigma^{2}\right)$ in (5) implies that a probability distribution over $l$-space for a given $x$ is expressed by [24]

$$
p(l x)=\left(2 \pi \sigma^{2}\right)^{-t / 2} \exp \left\{\frac{-1}{2 \sigma^{2}}\|l-W x-\mu\|^{2}\right\},
$$

with a Gaussian over latent variables defined by

$$
p(x)=(2 \pi)^{-q / 2} \exp \left\{\frac{-1}{2} \cdot x^{T} \cdot x\right\} .
$$

Here, in Case 3, $q=1$; so

$$
p(x)=(2 \pi)^{-1 / 2} \exp \left\{\frac{-1}{2} \cdot x^{T} \cdot x\right\}
$$

and marginal distribution of $l$ is in the form

$$
\begin{aligned}
& p(l)=\int p(l x) \cdot p(x) \cdot d x, \\
& p(x) \\
& \quad=(2 \pi)^{-q / 2}|C|^{-1 / 2} \exp \left\{\frac{-1}{2} \cdot(l-\mu)^{T} \cdot C^{-1}(l-\mu)\right\},
\end{aligned}
$$

where $q=1$; and the model covariance is

$$
C=\sigma^{2} I+W W^{T}
$$

Net transmitted power is $\sum_{i=1}^{n}\left|f_{i}\right|^{2}$ and the received signal power is $\left|\sum_{i=1}^{n} f_{i} \alpha_{i 1}\right|^{2}$. Hence, for this case, the power allocation problem can be stated as

$$
\begin{array}{ll}
\operatorname{minimize} & \sum_{i=1}^{n}\left|f_{i}\right|^{2} \\
\text { subject to } & \frac{\left|\sum_{i=1}^{n} f_{i} \alpha_{i 1}\right|^{2}}{P_{N}} \geq \mathrm{SNR}_{\min } \\
& \left|\sum_{i=1}^{n} f_{i} \alpha_{i 1}\right|^{2} \geq \mathrm{SNR}_{\min } P_{N} \\
& \left|f_{i}\right|^{2}\left|\sum_{i=1}^{n} \alpha_{i 1}\right|^{2} \geq \mathrm{SNR}_{\min } P_{N} .
\end{array}
$$

Applying Lagrangian multiplier technique for each node,

$$
\left|f_{i}\right|=\frac{\alpha_{i 1}}{\left|\sum_{i=1}^{n} \alpha_{i 1}\right|^{2}} \sqrt{\operatorname{SNR}_{\min } P_{N}} .
$$

Resulting link cost by the use of cooperation $C_{m-p}\left(S, r_{1}\right)$ defined as the optimal net power is therefore expressed as

$$
C_{m-p}\left(S, r_{1}\right)=\bar{P}_{T}=\frac{1}{\sum_{i=1}^{n}\left(\alpha_{i 1}^{2} /\left(\mathrm{SNR}_{\min } P_{N}\right)\right)}
$$

Case 4 (\{multipoint-to-multipoint link\}, i.e., $(n>1, m>1))$. In this case, $S_{n}=\left\{s_{1}, s_{2}, \ldots, s_{n}\right\}$, and $R_{m}=\left\{r_{1}, r_{2}, \ldots, r_{m}\right\}$ as shown in Figure 5. This is the MIMO case which helps implementation in cooperative reception and transmission of data among group sensors. It is assumed that all sensor nodes are working in half-duplex mode. This case is a combination of working formulations of Cases 2 and 3. In Case 2, multiple nodes are transmitting; thus the link cost in (3) applies for $n$ transmitters and $m$ receivers. Moreover, the technique of PCA is applicable for a group of $v$-dimensional data vectors $\left\{v_{t}\right\}, t \in\{1,2, \ldots, T\}$. Similarly, in Case 3, we considered a single receiver node with $q=1$; however here the receiving nodes are more than 1 in distinct dimensions; thus $q<t$ and (7) and (10) apply for any value of $q$, and the formulation of factor analysis applies. 


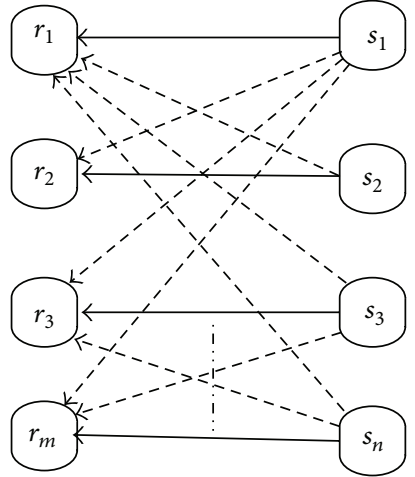

Figure 5: MIMO linkages.

\section{Attenuation, Propagation Delay, and Noise in UW Channel}

Simulation of UWSN communication links requires modeling the acoustic waves propagation in the scenario that a sensor node in UW struggles to forward data to another one. Sound propagates in the underwater environment at approximate speed of $c=1500 \mathrm{~m} / \mathrm{s}$. As a signal travels towards a node, its energy dissipates and it is distorted by noise. For UWA links, both link distance $d$ and signal frequency $f$ have dependency on attenuation denoted by $A(d, f)$. Hence, for a generated signal with low bandwidth, centered around frequency $f$ with power of unity, the received signal has an SNR expressed as $\rho(d, f)[15]$.

Underwater channel is influenced by both spreading and absorption losses causing attenuation to a large extent. For a separation of $d(\mathrm{~km})$ for a source and destination at a frequency $f(\mathrm{kHz})$ and spreading coefficient $k$, the attenuation $A(d, f)$ is expressed by Urick [26] given as

$$
A(d, f)=A_{0} d^{k} a(f)^{d},
$$

where $A_{0}$ is called normalizing constant. $k$ is known as the spreading factor whose value is $k=1$ for shallow water propagation, $k=2$ for deep water propagation, and for practical spreading $k=1.5$. The absorption coefficient $a(f)$ is modeled by the Thorps formula as [27]

$$
\begin{aligned}
& 10 \log a(f)=\frac{0.11 f^{2}}{1+f^{2}}+\frac{44 f^{2}}{4200+f}+\frac{2.75 f^{2}}{10^{4}}+0.003 \\
& \quad(\text { for } f>0.4)[\mathrm{dB} / \mathrm{km}], \\
& 10 \log a(f)=0.002+\frac{0.11 f}{1+f}+0.011 f \\
& \quad(\text { for } f<0.4)[\mathrm{dB} / \mathrm{km}] .
\end{aligned}
$$

In the absence of site-specific noises, receiver is influenced only by ambient noises, with overall Power Spectral Density (PSD) in terms of $\mathrm{dB}$ relative to $\mu \mathrm{Pa}$ in $\mathrm{kHz}$. The background noise in UW has various sources according to frequency and location, like turbulence $\left(N_{t}\right)$, shipping $\left(N_{s}\right)$, waves $\left(N_{w}\right)$, and thermal noise $\left(N_{\text {th }}\right)$. These noise effects are modeled by Gaussian statistics. The PSD of these ambient noises as described in [27] is

$$
N(f)=N_{t}(f)+N_{s}(f)+N_{w}(f)+N_{\mathrm{th}}(f),
$$

where

$$
\begin{aligned}
& 10 \log N_{t}(f)= 17-30 \log f, \\
& 10 \log N_{s}(f)= 40+20(s-0.5)+26 \log f \\
&-60 \log (f+0.03), \\
& 10 \log N_{w}(f)=50+7.5 \sqrt{w}+20 \log f \\
&-40 \log (f+0.4), \\
& 10 \log N_{\text {th }}(f)=-15+20 \log f,
\end{aligned}
$$

where $s$ is shipping activity factor, whose value ranges between 0 and 1 for low and high activity, respectively; and $w$ is the wind velocity ranging from 0 to $10 \mathrm{~m} / \mathrm{s}$.

\section{SNR in UW Acoustic Channel}

The SNR of a generated underwater signal having transmit power of unity $\widehat{p}(t)$ [watts] at the receiver is expressed by

$$
\operatorname{SNR}(d, f)=\rho(d, f)=\mathrm{SL}-A(d, f)-N(f)-\mathrm{DI},
$$

where $A(d, f)$ is attenuation and $N(f)[\mathrm{W} / \mathrm{Hz}]$ is the noise Power Spectral Density (PSD) given in (15) and (18), respectively. The noise model is the same as considered in (6) with variance $\sigma^{2}$ which is utilized here.

Directivity index is DI $=0$ if we assume an omnidirectional antenna. Source level SL $=20 \log I / 1 \mu \mathrm{Pa}$, if $I$ is intensity at unity distance from a source node in watt $/ \mathrm{m}^{2}$, given by

$$
I=\frac{\widehat{p}(t)}{2 \pi H}
$$

in which $H$ is the water depth. Signals travelling in underwater channels $\left(T_{d}\right)$ normally experience frequency and pathlosses which are much complicated than radio channels and are modeled as [27]

$$
T_{d}=10 \log _{10} d+10^{-3} a(f) d,
$$

where $a(f)$ is given in (16). First term of (24) expresses the power consumption of signals propagating from source to receiver in wireless channels. Second term is the absorption of propagating wave power due to acoustic waves [27]. Figure 6 shows the schematic flow chart for the SPARCO protocol.

\section{Outage Formulation in UWA Channel}

The noises in UW follow Gaussian distribution and the channel is stable for some period known as the coherence time. 


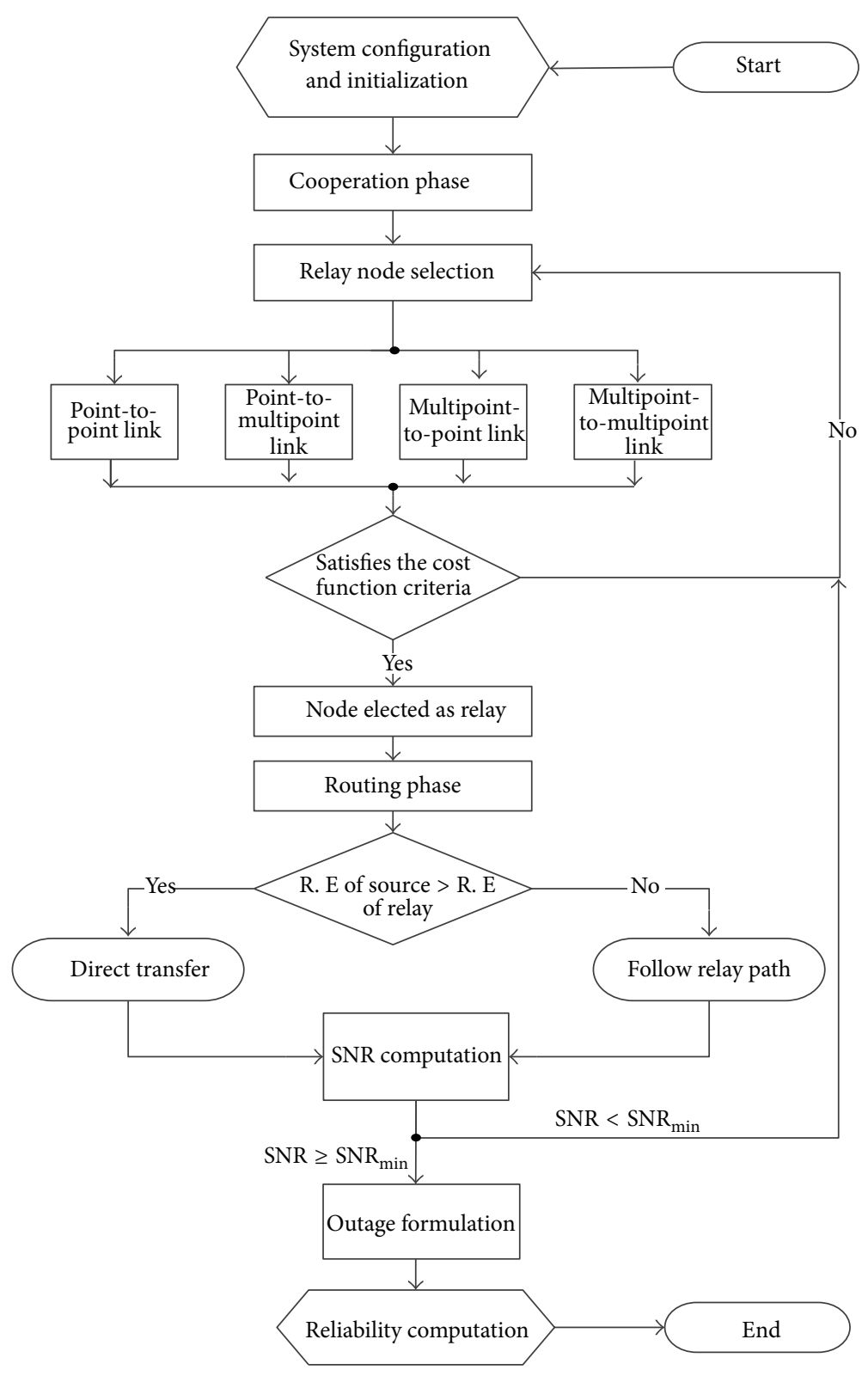

FIGURE 6: Flow chart of SPARCO protocol.

The capacity of a channel with infinite bandwidth determines the upper limit on the maximum information which can be communicated over a channel successfully. Shannon-Hartley theorem [27] expresses this by stating that "A communication system has a maximum information transfer rate $C$ known as the channel capacity. If the information rate $R$ is less than $C$, then we can expect arbitrarily small error probabilities using intelligent coding techniques. To get minimum error probabilities, the encoder has to work on longer blocks of signal data. This entails longer delays and higher computational requirements." Thus,

$$
C(d, \rho)=B \log _{2}(1+\rho(d, f))
$$

where $C(d, \rho)$ [bits/sec] is the channel capacity depending on both frequency and distance, whose expression makes intuitive sense:

(1) When the bandwidth of a channel rises, then we can make rapid variations in the information signal, which increases the information rate.

(2) When the value of $S / N$ rises, we can raise the information rate while still preventing errors due to noise.

(3) If noise is absent, $S / N$ approaches $\infty$ and a maximum information transfer is possible independent of bandwidth. 
Hence there is a trade-off bandwidth for SNR. However, as $B \rightarrow \infty$, channel capacity does not tend to infinity because, with a rise in bandwidth, the noise power also increases.

Let the information transfer rate at each sensor be $R$ [bits/sec]; then the signal is expected to be forwarded successfully on lossy channels, if the capacity is equal to or more than the transfer rate; that is,

$$
C(d, \rho) \geq R
$$

These criteria assess the quality of incoming signal at the destination. This helps us to approximate the efficiency of a link in wireless systems without any variations in detection, decoding, or complex coding mechanisms. Contrary to (26), outage event can take place when the data transfer rate $R$ goes more than $C$; that is,

$$
\text { Outage }=C(d, \rho)<R \text {. }
$$

An error is said to take place when the channel goes in outage or a decoding error occurs. The probability of error is approximately zero if the channel is not in outage. Therefore, the outage probability $P_{\text {outage }}$ is expressed as

$$
\begin{aligned}
P_{\text {outage }} & =P\{C(d, \rho)<R\}, \\
P_{\text {outage }} & =P\left\{B \log _{2}(1+\rho(d, f))<R\right\}, \\
P_{\text {outage }} & =P\left\{\rho(d, f)<2^{(R / B)}-1\right\}, \\
P_{\text {outage }} & =P\left\{20 \log \left(\frac{\widehat{p}(t)}{2 \pi H}\right)-A(d, f)-N(f)\right. \\
\left.<2^{(R / B)}-1\right\}, & \\
P_{\text {outage }}=P\left\{\log \left(\frac{\widehat{p}(t)}{2 \pi H}\right)\right. & \left.2^{(R / B)}+A(d, f)+N(f)-1\right\}, \\
\left.<\frac{20}{2 \pi}\right\} & =P\{\widehat{p}(t)<2 \pi H \text { anti } \\
P_{\text {outage }} & \left.=P\left(\frac{2^{(R / B)}+A(d, f)+N(f)-1}{20}\right)\right\} .
\end{aligned}
$$

Equation (33) clearly indicates that the outage probability at any instant is totally dependent on the depth of the ocean and the attenuation and noise factors occurring in ocean currents. In terms of SNR and ignoring the (-1) term, (33) can be expressed as

$$
\begin{aligned}
& P_{\text {outage }} \\
& \qquad=P\left\{\widehat{p}(t)<2 \pi H \exp \left(\frac{2^{(R / B)}+\mathrm{SL}+\rho(d, f)}{20}\right)\right\} .
\end{aligned}
$$

\section{Reliability in UWA Channel}

With the passage of time and advancement in technology, there are lots of methods that are used to avoid the loss of data when the channel is in outages like employing ARQ protocols, obtaining information from the transmitter side channel, or coding for a longer period [28]. However, this study concentrates and focuses on the reliability of the link to be obtained through the use of routing by isolating the diversity obtaining issue, and the results can be associated with other diversity techniques. Different links combine to form hops and then these multiple-hop paths make a sequential combination of nodes which pass the information to one another and ultimately lead to the destination $D$ from a source $S$. That event will be deemed as a successful end-toend transmission in which all the packets or transmissions are successful and the probability of occurrence of an event is defined as End-to-End Reliability denoted by $\mathfrak{R}$ [29]. Hence, $\mathfrak{R}$ can be written as

$$
\begin{aligned}
& \Re=1-P_{\text {outage }} \\
& \Re=1 \\
& \quad-\left\{\widehat{p}(t)<2 \pi H \exp \left(\frac{2^{(R / B)}+\mathrm{SL}+\rho(d, f)}{20}\right)\right\} .
\end{aligned}
$$

According to this expression, $\mathfrak{R}$ is a monotonically decreasing function and establishes the result for a point-to-point link. It is dependent on the depth of the water, channel state, and the distance between two nodes. The net reliability for the entire end-to-end path can be computed from (36) as $\mathfrak{R}=1-\left\{\sum_{i=1}^{n}\left(2 \pi H_{i} \exp \left(\left(2^{(R / B)}+\mathrm{SL}+\rho(d, f)_{i}\right) / 20\right)\right)\right\}$.

The maximum reliability route is the route that minimizes this sum and the maximum amount of power that can be spent in relaying the information from $S$ to $D$ is limited to the summation of SNR for individual nodes as given in (14).

\section{Performance Evaluation of SPARCO}

Major metrics of performance for all compared protocols are defined as follows.

\subsection{Performance Metrics}

9.1.1. Stability Period. The total time interval between the initiation of network till the death of first node is called stability period.

9.1.2. Residual Energy. It is defined as the difference of the initial applied energy and that of the utilized energy by the nodes during network operation.

9.1.3. Network Lifetime. The total time taken by the network operation is called network lifetime.

9.1.4. Throughput. Throughput is the term which shows the total number of successfully received packets at the sink. 
9.1.5. Delay Spread. The measure of density or abundance of the multipath of a communication channel is known as delay spread. It is calculated by taking the difference between the latest multipath component and the earliest multipath component of the received signal in network operation.

9.1.6. Path-Loss. Measured in decibels $(\mathrm{dB})$ is the difference of the transmitted and received powers of transmitting nodes and receiving nodes, respectively.

9.2. Results and Discussions. Existing schemes, CoDBR, CogCoop, iAMCTD, and Coop ( $\mathrm{Re}$ and $\mathrm{dth}$ ), are used as benchmark to evaluate and analyze SPARCO performance. Nodes are randomly deployed for every simulated technique. Utilizing multiple-sink model of conventional methods having 10 sinks deployed on the water surface, 225 nodes are deployed randomly in a network field of $500 \mathrm{~m} \times 500 \mathrm{~m}$ with 4 courier nodes acting as relays. The alive nodes in the network transmit threshold-based data to another neighbor node which in turn forwards it to another neighbor. There is a proper coordination between the nodes that all share proper physical parameters, notably weight and depth threshold, with the neighbor node to keep informed with the fluctuating circumstances of the network. Nodes calculate their distances from their neighbors after fixed intervals. Sensors forward their information to the upper layer using cooperation of neighboring sensors till that information reaches the sink. The sink supervises the depth thresholds and adaptive mobility of cooperating sensors. The introduction of depth thresholds and cooperation makes SPARCO scheme a feasible application for data-critical situations.

Figure 7 shows a comparison of the stability period of SPARCO, CoDBR, iAMCTD, Coop (Re and dth), and CogCoop with respect to network lifetime. It is obvious from the figure that due to the reason of maintaining lower pathloss and neglecting the unnecessary data forwarding results in higher stability than the other four schemes. In the time interval after which the nodes start dying, in the simulations of 10,000 seconds, the initial or first node in case of SPARCO dies at 4290th second which is more extended than the other four. This increases the stability duration such that, in CoDBR, first node dies after 961st second; in iAMCTD it dies after 3185th second; in Cog-Coop it dies after 1857th second; and in Coop (Re and dth) it dies after 961st second. In other words, we can say that the instability period starts after around 4300 seconds, after which the end-to-end delay decreases very slowly. Cooperative nodes play an important role in SPARCO, Coop (Re and dth), CoDBR, and Cog-Coop by the introduction of cooperation scheme, because these nodes distribute and share the load of data forwarding which results in achievement of load balancing, hence increasing the stability period. Network lifetime of iAMCTD gets increased compared to CoDBR, as there is a slow raise in energy consumption. As the network becomes sparse slowly, number of neighbor nodes falls suddenly in CoDBR which causes the instability in the network. iAMCTD considers two forwarding attributes, depth and residual energy, which causes a trade-off between network stability and path-loss. This

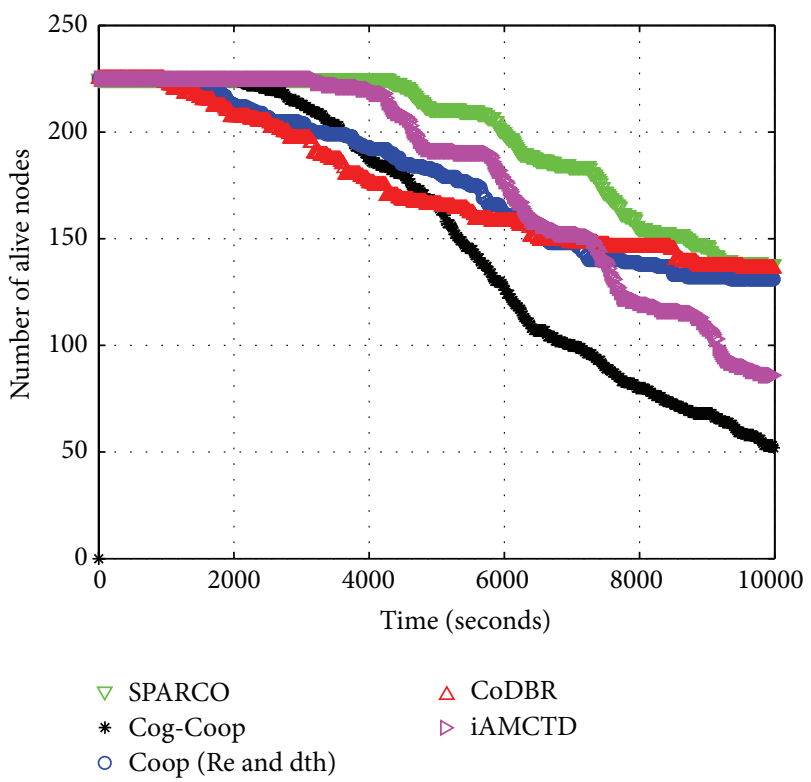

Figure 7: Alive nodes versus network lifetime.

does not make it suitable for reactive applications. Network stability of iAMCTD is raised in comparison to CoDBR, due to lower throughput by responsive network.

Cooperation between nodes causes load balancing in both SAPRCO and Coop (Re and dth). In our proposed protocol, utility of Thorps energy model identifies the net channel losses, which are quite needful for selection of appropriate data forwarding. The consideration of channel losses is absent in the Co-DBR scheme. Raise in stability period of SPARCO also confirms lowering of redundant transmissions. The stability period of iAMCTD is better than CoDBR and Coop (Re and dth); however, the network energy consumption is greater in CoDBR. In iAMCTD and Coop ( Re and $\mathrm{dth}$ ), the source nodes are transmitting the date to nexthop neighbor nodes only, whereas CoDBR is utilizing source node along with two relay nodes to transmit data to the next hop. In CoDBR, the stability period finishes too suddenly due to prioritization of residual energy in selection of reasonable neighbor nodes, which causes inefficient instability period. Table 1 indicates a numerical comparison of all the four compared protocols in terms of alive nodes after equal intervals. The table shows that as the stability periods of Co-DBR and Coop ( $\mathrm{Re}$ and $\mathrm{dth}$ ) are the least, hence if we keep them as reference, then the percentage improvements in other schemes are shown numerically with regard to these protocols.

Figure 8 shows the graphical representation of the difference between the energy consumption of iAMCTD (existing noncooperative schemes), CoDBR and Cog-Coop (existing cooperative schemes), and SPARCO. As the graph shows, the energy is more efficiently utilized in SPARCO than the existing schemes due to efficient forwading of data with the help of neighbor nodes and load balancing is ultimately achieved. Another reason for the efficiency is that energy consumption is improved with the help of effective weight implementation. As SPARCO's main concern is with those 
TABLE 1: Alive nodes available after specified intervals in seconds.

\begin{tabular}{lcccccccc}
\hline Protocol & First node dies at & Efficiency in percentage & 1000 & 2000 & 4000 & 6000 & 8000 & 10000 \\
\hline CoDBR & 961 & 100 & 225 & 207 & 176 & 158 & 146 & 136 \\
iAMCTD & 3185 & 331.4 & 225 & 225 & 220 & 177 & 120 & 86 \\
Cog-Coop & 1857 & 193.2 & 225 & 225 & 187 & 126 & 80 & 52 \\
Coop (Re and dth) & 961 & 100 & 225 & 213 & 193 & 162 & 138 & 131 \\
SPARCO & 4290 & 446.4 & 225 & 225 & 225 & 200 & 155 & 138 \\
\hline
\end{tabular}

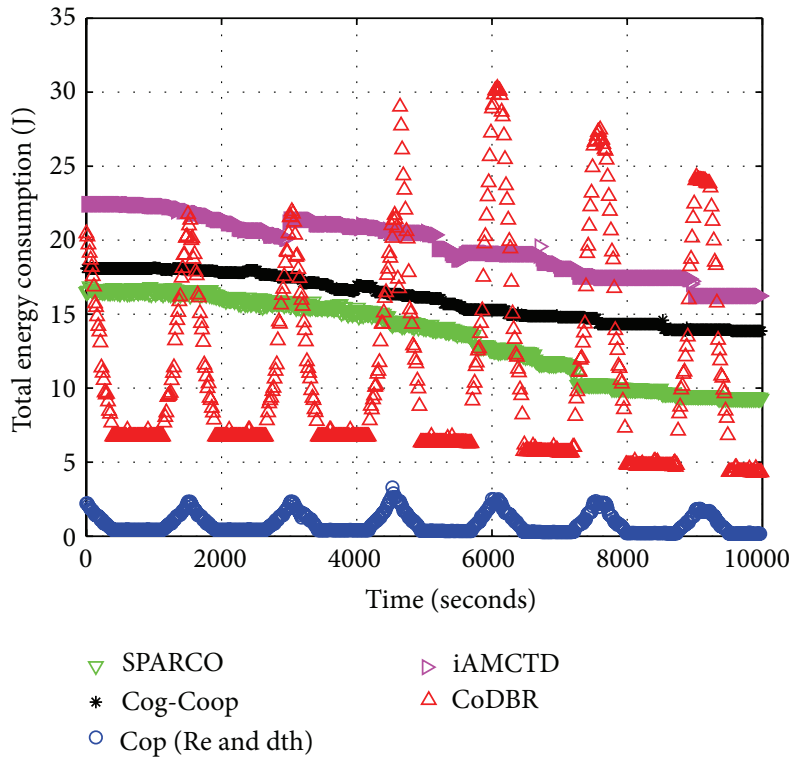

FIGURE 8: Total energy consumption versus network lifetime.

applications which are time bound, therefore it has to be efficient, and for the purpose of which it utilizes cooperation and depth difference between data forwarders in the network to solve the issue of energy consumption.

In iAMCTD, the larger distance between the neighboring nodes makes them consume high energy, while, in other existing schemes like CoDBR, the frequent switching to select high energy nodes makes them consume higher energy than other existing techniques making it least efficient. The stability period of Cog-Coop is extended compred to CoDBR and iAMCTD; however, the network energy consumption is greater in Cog-Coop. The cooperative transmission scheme Cog-Coop is consuming approximately three times more energy than CoDBR and iAMCTD in stable region. As the nodes start to die after 3000 seconds, the total energy consumption tends to increase more sharply. In Cog-Coop and iAMCTD, the source nodes are transmitting the date to next hop neighbor nodes only, whereas CoDBR is utilizing source node along with two relay nodes to transmit data to the next hop. In Coop (Re and dth), there is a sudden increase in network energy consumption during the initial rounds as all nodes become active and perform the routing process. Later on, energy consumption decreases because nodes fail to find relay nodes due to reduction in network density. Hence, chances of cooperative routing being performed by any source node are reduced which in turn reduces energy consumption. Table 2 illustrates a comparison of residual energy left, in percentage, of all the five compared protocols after equal intervals which clearly shows that in SPARCO the value of residual energy drop is the least among the four. The table shows that the maximum residual energy drop is in Cog-Coop and if we consider its drop to be maximum, then the other schemes show improvements in reference to this scheme. The maximum efficiency in percentage is shown by SPARCO whose averaged drop is $65.2 \%$ in comparison to Cog-Coop which is assumed to be $100 \%$.

Packet delivery ratio (PDR) is the ratio of data packets received at receiver end to those generated by the source which is the throughput in other words. The comparative analysis of all the four schemes under study based on PDR comparison is illustrated in Figure 9. As we can see, the drop in the PDR in SPARCO is much less as compared to other schemes. Network lifetime of Coop (Re and dth) gets lowered but the delivery ratios of iAMCTD and CoDBR show similar plots. The delivery ratio in Cog-Coop is improved compared to that of SPARCO, although both schemes are utlizing cooperation. This is due to the fact that Cog-Coop utilizes only two relay nodes whereas SPARCO utilizes multiple relay nodes and considers SNR in the links. Traffic is flooded from the source nodes in case of iAMCTD and CoDBR, when the total time between the source and destination in nodes is small, which ultimately results in lower PDR due to the increase in packet collision.

In SPACRO scheme instead of single path, a number of different multiple paths are used to forward the data and then they are combined at the receiver node. Due to this, the data which is sent in packets has higher chance of being successfully transmitted. A larger number of cooperating nodes are available for data forwarding whereas higher reliability can be achieved. The sudden drop in the delivery ratio of SPARCO is due to the fact that the relay nodes using cooperation consume more energy than normal nodes and hence die early, which creates instability in the network. On the other hand, the other two schemes are lagging behind in the reliability of forwarding packets because, in CoDBR, the distant propagation as well as multiple forwarding used results in lower PDR. In iAMCTD, the propagations remain stable due to the fact that both residual energy and depth are considered in the weight function computation which makes it better than CoDBR in terms of transmission loss. Coop (Re and dth) scheme shows a similar type of risefall behavior in case of PDR because the scheme does not consider the channel conditions as well as the SNR of the link and the throughput decreases due to quick fall in network density. Table 3 shows a numerical comparison of all the five 
TABLE 2: Residual energy drop in percent after equal intervals in seconds.

\begin{tabular}{lcccccc}
\hline Protocol & Averaged efficiency in percentage & 2000 & 4000 & 6000 & 8000 & 10000 \\
\hline CoDBR & 95.6 & 5.06 & 11.4 & 24 & 36.7 & 39.87 \\
iAMCTD & 88.1 & 3.65 & 15.8 & 22.7 & 29.12 \\
Cog-Coop & 100 & 2.65 & 17.8 & 25.52 & 36.35 \\
Coop (Re and dth) & 72.5 & 3.2 & 11.5 & 17.4 & 40.5 \\
SPARCO & 65.2 & 8 & 9.2 & 14.4 & 23 & 33.6 \\
\hline
\end{tabular}

TABLE 3: Packet delivery ratio after equal intervals in seconds.

\begin{tabular}{lccccc}
\hline Protocol & Averaged efficiency in percentage & 2000 & 4000 & 6000 & 8000 \\
\hline CoDBR & 60 & 0.97 & 0.76 & 0.57 & 0.36 \\
iAMCTD & 66.6 & 1 & 0.93 & 0.65 & 0.41 \\
Cog-Coop & 92.6 & 1 & 1 & 1 & 0.34 \\
Coop (Re and dth) & 68.8 & 1 & 0.93 & 0.63 & 0.34 \\
SPARCO & 94 & 1 & 1 & 0.55 & 0.68 \\
\hline
\end{tabular}

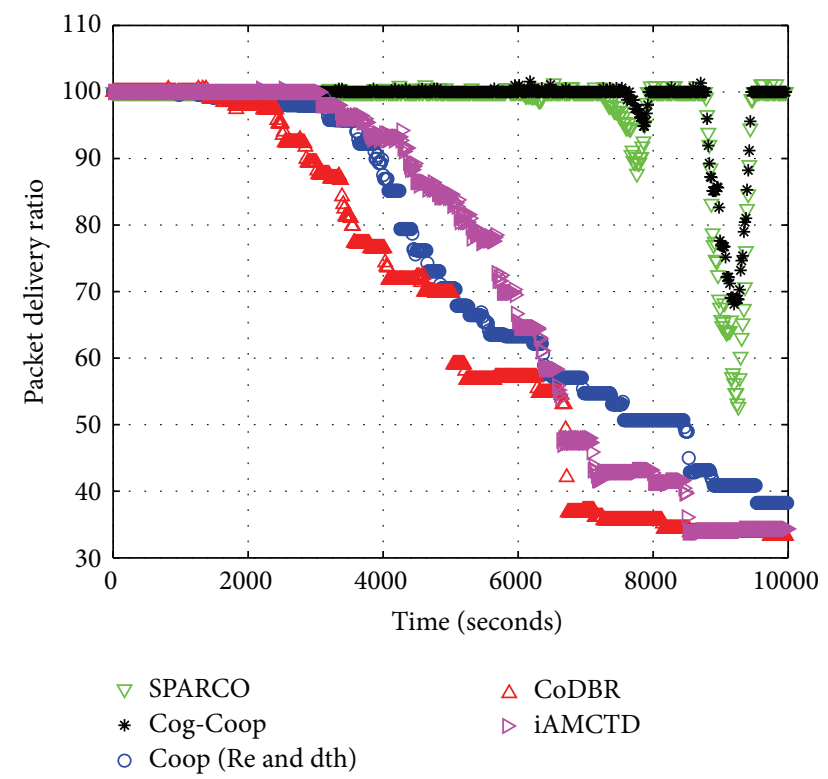

FIGURE 9: Packet delivery ratio versus network lifetime.

compared protocols in terms of PDR after equal number of intervals. The table also highlights the averaged efficiency of all the compared schemes in terms of PDR and SPARCO shows an averaged efficiency of $94 \%$.

Figure 10 further illustrates that the path-loss of the network in CoDBR, Coop (Re and dth), Cog-Coop, and iAMCTD is much higher than SPARCO because of prioritizing SNR in its modeling. The plots very clearly indicate that the effective use of relay nodes in SPARCO and iAMCTD outperforms the other three schemes. Distant transmissions are preferred in both Cog-Coop and CoDBR, while, on the contrary to the existing schemes, Uricks model and Thorps attenuation model for UWA are utilized in SPARCO and iAMCTD to trace out and measure the total loss in transmission during data transfer between the source and the destination. It takes into account transmission frequency,

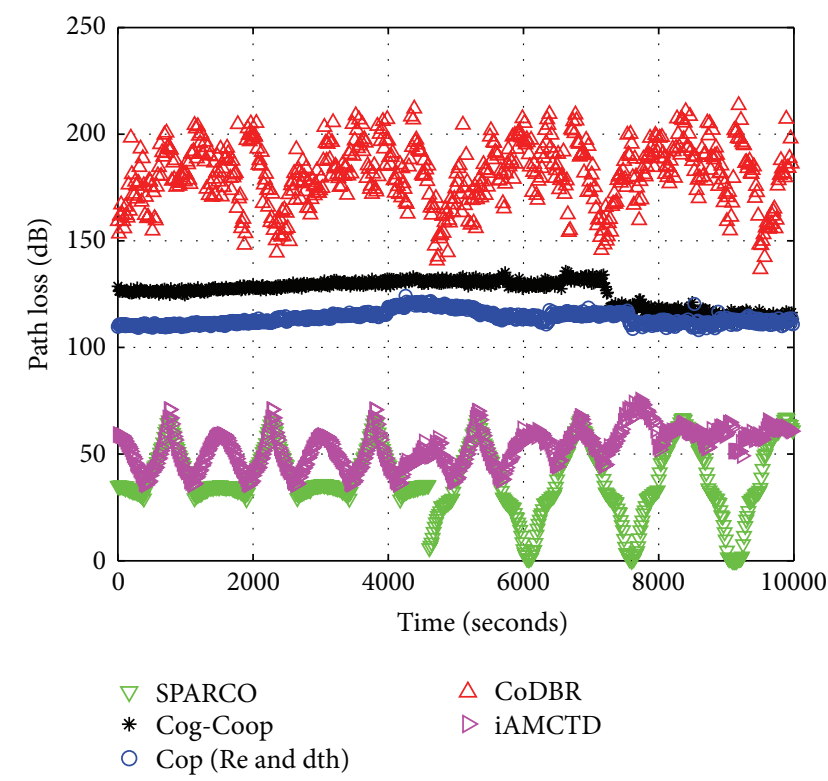

Figure 10: Path-loss of network versus network lifetime.

bandwidth efficiency, and noise effects which scrutinize the signal quality during data forwarding. CoDBR and Coop (Re and dth) are cooperative schemes but do not consider any noise factors and transmission losses as in SPARCO; that is why their performance is much less compared to SPARCO.

Cog-Coop, due to the high network density, shows less losses in starting phases of node deployment. As the network scatters, its losses increase which haunts the network performance ultimately resulting in high packet loss during its transmission. In iAMCTD, channel loss conditions are better than CoDBR and Coop ( $\mathrm{Re}$ and dth), as its cost computation considers depth as well as residual energy of forwarding nodes; hence, the propagations remain stable. SPARCO again gives good results compared to the other schemes as its SNR calculation takes into account both depth and residual energy of the relay nodes as well as the utilization 
TABLE 4: Path loss (dB) after equal intervals in seconds.

\begin{tabular}{lccccc}
\hline Protocol & Averaged efficiency in percentage & 2000 & 4000 & 6000 & 8000 \\
\hline CoDBR & 21.4 & 197 & 196 & 196 & 193 \\
iAMCTD & 81.4 & 40 & 43 & 58 & 53 \\
Cog-Coop & 33.3 & 129 & 130 & 128 & 119 \\
Coop (Re and dth) & 36.5 & 112 & 119 & 115 & 116 \\
SPARCO & 100 & 39 & 43 & 26 & 111 \\
\hline
\end{tabular}

of cooperation scheme, which ultimately results in more stable propagations. In SPARCO, we have utilized Uricks model and Thorps attenuation model for UW environment to calculate the transmission loss in packet forwarding. These models determine the effect of path-loss on transmission frequency, bandwidth efficiency, and noise density during data transmission. Table 4 indicates a numerical comparison of all the four compared protocols in terms of path-loss after equal intervals traversed. The table shows that the efficiency of our scheme SPARCO is maximum in comparison to other schemes in terms of path-loss and the scheme Co-DBR shows the minimum efficiency that is approximately $21 \%$.

Figure 11 shows a comparison of dead nodes in case of SPARCO with those of CoDBR, Coop (Re and dth), CogCoop, and iAMCTD. The figure shows the time interval after which the nodes start dying. In the simulations of 10,000 seconds, the initial or first node in SPARCO dies being in operation after 4300th second which is greater than the other four schemes. This increased the stability duration such that, in CoDBR, nodes start dying after 970th second; in iAMCTD they start dying after 1700th second; in Coop ( $\mathrm{Re}$ and $\mathrm{dth}$ ) they start dying after 1000 seconds; and in Cog-Coop the nodes start dying after around 1900 seconds. Cooperative nodes play an important role by the introduction of cooperation scheme in both SPARCO and Coop (Re and $\mathrm{dth}$ ), because relay nodes distribute and share the load of data forwarding which results in achievement of load balancing, hence increasing the stability period.

Due to the fact that in iAMCTD there is a gradual increase in network energy consumption, its stability period is greater than CoDBR and Coop ( $\mathrm{Re}$ and $\mathrm{dth}$ ). The primary reason which causes network instability in CoDBR is that as the network becomes sparse, the number of neighbor nodes decreases quickly. As discussed, there are two forwarding attributes in iAMCTD which are depth and residual energy. This results in the trade-off between path-loss of packets and network lifetime which is not appropriate for reactive applications. During the instability period of iAMCTD and Coop (Re and dth), network slowly gets sparse creating load on high energy nodes, while the number of neighbors is handled by variations in depth threshold. The stability period of Cog-Coop is less than SPARCO because the nodes are consuming three times more energy than SPARCO. Lifetime of SPARCO is increased due to lower throughput by reactive network. In the proposed schemes, Thorps energy models help to analyze specifically and in detail the channel losses, which is very useful in reactive networks for data forwarding. The redundant transmissions are also reduced due to the increase in stability period. Table 5 illustrates the comparative

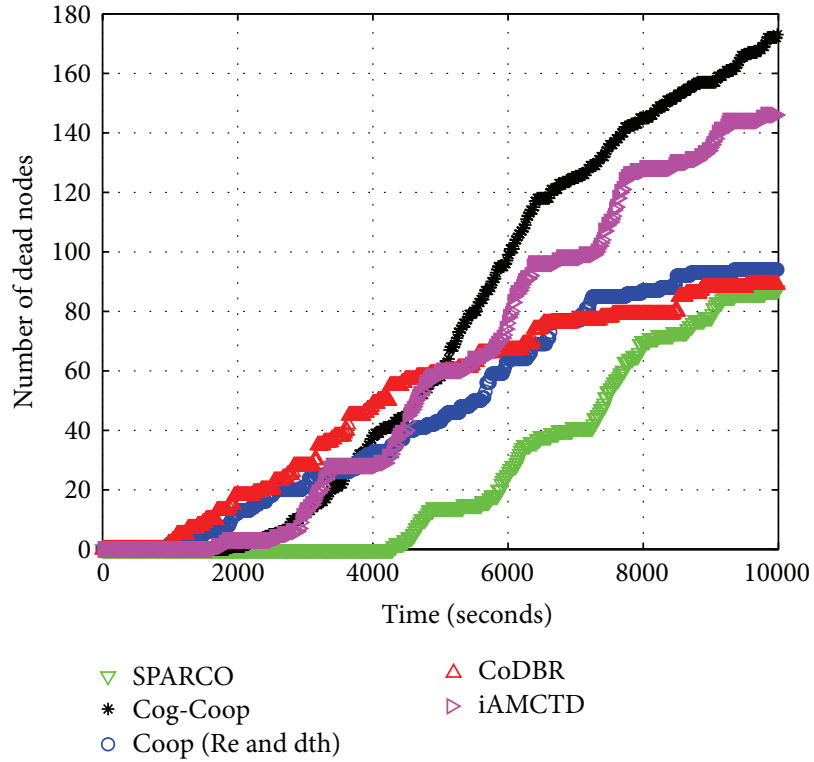

FIgURE 11: Dead nodes versus network lifetime.

analysis of all the five schemes under study in terms of the total dead nodes after equal intervals of time. The difference in the table clearly shows that SPARCO has least number of dead nodes compared to its counterparts.

End-to-end delay with respect to five schemes is depicted in Figure 12. Their comparisons show that the delay in case of SPARCO is lower than the previous schemes due to least transmission distances between the sensors in both sparse and dense situations. Coop ( $R e$ and $d t h$ ) has the highest delay compared to the other four schemes because it is utilizing relay nodes in every data transfer and also does not consider any losses present in the UW medium. In CoDBR, delay is much higher in the beginning due to distant data transmission. It slowly gets lowered with the sparseness of the network and the network causes data transferring at least distance. In iAMCTD, the end-to-end delay is better than the previous schemes due to the load balancing of both threshold variations and weight functions. But, in SPARCO, there is a minimum time lag because of the consideration of SNR, difference in depths of sender and receiver sensors, and introduction of cooperation. iAMCTD and CoDBR transfer data with least hops; however the attenuated path raises the data loss at receiver, and packets need to be reforwarded. This intensifies the packet delay. While all the four protocols are focusing on channel estimation, data packets are forwarded with better reliability, resulting in 
TABLE 5: Dead nodes available after specified intervals in seconds.

\begin{tabular}{lcccccccc}
\hline Protocol & First node dies at & Efficiency in percentage & 1000 & 2000 & 4000 & 6000 & 8000 & 10000 \\
\hline CoDBR & 961 & 100 & 2 & 18 & 42 & 55 & 68 & 85 \\
iAMCTD & 3185 & 331.4 & Zero & 3 & 28 & 78 & 127 & 147 \\
Cog-Coop & 1857 & 193.2 & Zero & 1 & 38 & 99 & 145 & 173 \\
Coop (Re and dth) & 961 & 100 & 2 & 13 & 32 & 63 & 87 & 94 \\
SPARCO & 4290 & 446.4 & Zero & Zero & Zero & 25 & 70 & 87 \\
\hline
\end{tabular}

TABLE 6: End-to-end delay after equal intervals in seconds.

\begin{tabular}{lcccccc}
\hline Protocol & Averaged efficiency in percentage & 2000 & 4000 & 6000 & 8000 & 10000 \\
\hline CoDBR & 50.8 & 0.08 & 0.10 & 0.19 & 0.10 & 0.13 \\
iAMCTD & 22.9 & 0.05 & 0.06 & 0.05 & 0.05 \\
Cog-Coop & 63.55 & 0.13 & 0.16 & 0.17 & 0.15 \\
Coop (Re and dth) & 100 & 0.24 & 0.24 & 0.21 & 0.24 \\
SPARCO & 45.7 & 0.07 & 0.09 & 0.16 & 0.14 \\
\hline
\end{tabular}

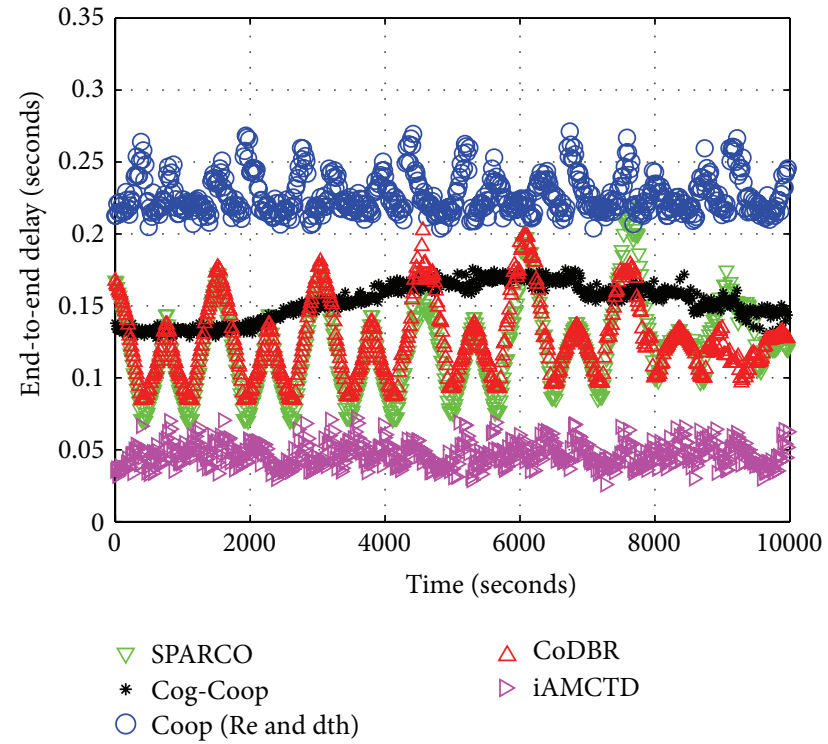

FIGURE 12: End-to-end delay versus network lifetime.

lower redundancy, especially in cooperative schemes Coop (Re and dth), CoDBR, and Cog-Coop. The packets, hence, reach the destination with much reduced delay. Table 6 indicates a numerical comparison of all the five compared schemes in terms of delay. It also highlights the percentage of efficiency of all the compared schemes with the maximum delay achieved by Coop (Re and dth) which is assumed to be $100 \%$ and all other improvements or drops in delays are expressed with reference to it.

Figure 13 shows the outage probability of proposed SPARCO scheme with a number of cooperative nodes acting as relays. These plots help in system design because outage probability is an important QoS parameter, and they allow us to find the optimal number of cooperative nodes. For this simulation, we set the transmission rate equal to 1 as outage probability is dependent on the transmission rate. The blue line in Figure 12 shows the variation in outage probability

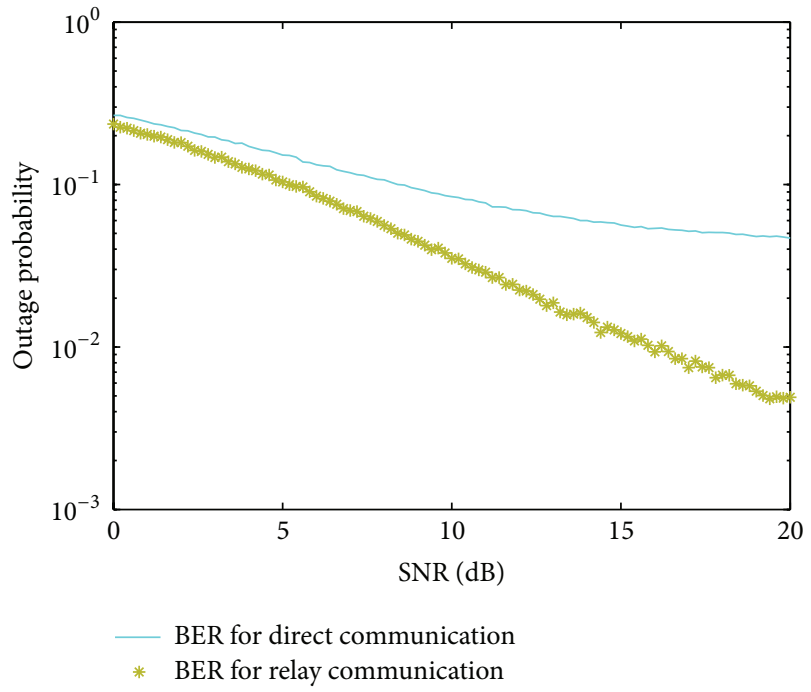

FIGURE 13: Outage probability versus SNR. Outage probability versus SNR for direct and relay communication.

for direct communication between a transmitting node and receiving node and no relay is utilized. On the other hand, variation of outage probability with that of SNR for a fully cooperative environment is shown by the green line.

The plots in Figure 13 clearly indicate that, for lower values of SNR till $6 \mathrm{~dB}$, there is no major difference in the two plots. However, beyond this value, we find a remarkable improvement in outage probability for cooperative or relay communication in contrast to direct communication. This is due to the fact that, with the increase in SNR value, more relay nodes take part in data transfers rather than direct communication in order to combat the varying underwater channel losses. Hence, we deduce that AF scheme can provide complete diversity such that the diversity order which utilizes outage probability as a performance measure is the net number of cooperating nodes in the network. This shows that the best number of cooperating sensors is in fact a complex 
TABLE 7: Outage probability for direct versus relay communication after equal intervals of SNR.

\begin{tabular}{lccccc}
\hline $\begin{array}{l}\text { Type of data } \\
\text { transfer }\end{array}$ & $\begin{array}{c}\text { BER at SNR } \\
0 \mathrm{~dB}\end{array}$ & BER at SNR $5 \mathrm{~dB}$ & $\begin{array}{c}\text { BER at SNR } \\
10 \mathrm{~dB}\end{array}$ & $\begin{array}{c}\text { BER at SNR } \\
15 \mathrm{~dB}\end{array}$ & $\begin{array}{c}\text { BER at SNR } \\
20 \mathrm{~dB}\end{array}$ \\
\hline Direct & 0.125 & 0.067 & 0.05 & 0.03 & 0.28 \\
Relay & 0.125 & 0.05 & 0.02 & 0.01 & 0.004 \\
\hline
\end{tabular}

function of SNR and the cooperative diversity method in use. Table 7 shows the comparison of the direct data transfer versus the relay utilized data transfer after equal intervals of SNR. The table clearly shows that the outage probability reduces significantly with SNR increase in case of relay transfer comparative to direct transfer.

9.3. Performance with Trade-Offs. In SPARCO, improvement in delay is targeted at the cost of time lag. This end-to-end delay in SPARCO is improved as compared to iAMCTD and CoDBR, but at the cost of possible time lag due to consideration of SNR and cooperation mechanism. In CoDBR, delay is improved at the cost of repeated transmissions. The delay in CoDBR is much higher in initial stages due to distant data forwarding but at the cost of redundant transmissions because the attenuated underwater channel increases packet loss at the receiving end. In AMCTD, delay is improved at the cost of energy depletion. The delay in iAMCTD is better than CoDBR as variations in depth threshold as well as weight functions perform load balancing, however, at the cost of abrupt energy depletion of the nodes. End-to-end delay in Coop ( $\mathrm{Re}$ and $\mathrm{dth}$ ) is improved but at the cost of energy utilization and transmission loss.

In SPARCO, the stability period is improved at the cost of more forwarding nodes and energy consumption. The protocol enhances the network lifetime by avoiding the transmission of useless data and maintaining lower transmission loss, however, at the cost of utilization of relay nodes and proper selection of relay forwarding nodes. In SPARCO, the instability period starts after 4300 seconds, after which the PDR remains even; however total energy consumption decreases slowly. In iAMCTD, the stability period is achieved at the cost of transmission loss. In this protocol, depth and residual energy are the only two forwarding selecting variables used in the scheme. This consideration compromises between the transmission loss of packets and the network lifetime or is a kind of trade-off between them which is not appropriate for reactive application at all. During the instability period of iAMCTD, network slowly becomes sparse creating load on high energy nodes. In CoDBR, the stability period is improved at the cost of greater energy consumption. In Cog-Coop, the stability period improves but at the cost of energy consumption as it utilizes two relay nodes in the data forwarding and also the delay increases as the data has to flow through the relay nodes till the sink is the next hop. In Coop (Re and dth), the stability period is improved at the cost of delay and transmission loss.

In SPARCO, the path-loss reduces at the cost of stability period and the energy consumption as redundant transmissions between sender sensors and sink is raised due to the use of relay nodes. Figure 9 illustrates how SPARCO is better than other techniques in terms of medium path-loss because of giving preference to SNR in its computations. In CoDBR, multiple transmissions increase path-loss between sender node and the sink. We utilize Thorps attenuation model for UWA to formulate the transmission loss in packet transferring between a source and destination node through relay. It considers transmission frequency, bandwidth, and noise factors which scrutinize the signal quality during data transmission. Higher throughput in iAMCTD is achieved at the cost of redundant transmissions between nodes and sink. In iAMCTD, channel losses are better than CoDBR and CogCoop, as the cost function considers both residual energy and depth of transferring sensors; hence, the propagations remain stable. With the passage of time in later stages, the qualified forward nodes decrease in number due to which the network experiences the delay in the transmission as far as the packet loss in iAMCTD. In Coop (Re and dth), the path-loss is improved at cost of high delay.

In SPARCO, throughput is improved at the cost of time lag. The drop in PDR in SPARCO is lower than that of other schemes. Higher traffic is sent from a source as the interarrival time of data packets gets lowered. This raises the chances of packet collision resulting in a less PDR. SPARCO protocol enhances the probability of successful packet reception by forwarding data on various links and then aggregating at the receiver node. This improvement is achieved at the cost of higher energy consumption of the network as more nodes are involved in the data forwarding mechanism. In CoDBR, transmission loss improved at the cost of low PDR. In this protocol, higher transmission loss is achieved than the other two schemes as it utilizes distant propagations as well as multiple forwarding. iAMCTD achieves improvement in throughput at the cost of packet loss and delay. In iAMCTD, channel loss conditions are better than CoDBR, as the weight function considers depth and residual energy of active nodes; therefore the propagations remain stable. However, in later stages, the performance of iAMCTD slowly gets lowered with the decrement in qualified forwarders; therefore both the packet loss and delay increase but at the cost of drop in its throughput. Table 8 indicates the various performance parameters which are enhanced on the price which they have to pay, for the five compared protocols.

\section{Conclusion}

In this research, we have proposed SPARCO routing scheme to enhance the stability period and reduce the energy consumption of underwater networks. Introduction of cooperation and SNR improves the network lifetime, improves 
TABLE 8: Performance parameters with their trade-offs.

\begin{tabular}{|c|c|c|c|c|}
\hline Protocol & Advances achieved & Reference & Price to pay & Reference \\
\hline \multirow{4}{*}{ SPARCO } & Stability period extends & Figures 7 and 11 & More forwarding nodes and energy consumption & Figure 8 \\
\hline & Transmission loss declines & Figure 10 & Shorter stability period and energy consumption & Figures 8 and 11 \\
\hline & Throughput increases & Figure 9 & Time lag and energy consumption & Figure 8 \\
\hline & End-to-end delay improves & Figure 12 & Transmission loss & Figure 10 \\
\hline \multirow{4}{*}{ CoDBR } & Stability period extends & Figures 7 and 11 & Packet delivery ratio & Fig. 9 \\
\hline & Transmission loss declines & Figure 10 & Redundant transmissions and lesser stability period & Figures 7 and 11 \\
\hline & Throughput increases & Figure 9 & Transmission loss and delay & Figures 10 and 12 \\
\hline & End-to-end delay improves & Figure 12 & Sharp energy depletion & Figure 8 \\
\hline \multirow{4}{*}{ iAMCTD } & Stability period extends & Figures 7 and 11 & Extra forwarding nodes and energy consumption & Figure 8 \\
\hline & Transmission loss declines & Figure 10 & Packet delivery ratio and delay & Figures 9 and 12 \\
\hline & Throughput increases & Figure 9 & Transmission loss due to distant propagations & Figure 10 \\
\hline & End-to-end delay improves & Figure 12 & Transmission loss due to courier nodes & Figure 10 \\
\hline \multirow{4}{*}{ Cog-Coop } & Stability period extends & Figures 7 and 11 & Energy consumption due to two nodes relaying and delay & Figure 8 \\
\hline & Transmission loss declines & Figure 10 & Redundant transmissions and lesser stability period & Figures 7 and 11 \\
\hline & Throughput increases & Figure 9 & Transmission loss and delay & Figures 9 and 11 \\
\hline & End-to-end delay improves & Figure 12 & Sharp energy depletion & Figure 8 \\
\hline \multirow{4}{*}{$\begin{array}{l}\text { Coop (Re } \\
\text { and dth) }\end{array}$} & Stability period extends & Figures 7 and 11 & Redundant transmissions and packet delivery ratio & Figure 9 \\
\hline & Transmission loss declines & Figure 10 & End-to-end delay & Figure 12 \\
\hline & Throughput increases & Figure 9 & Transmission loss and greater energy consumption & Figures 8 and 10 \\
\hline & End-to-end delay improves & Figure 12 & Transmission loss due to lag of SNR & Figure 10 \\
\hline
\end{tabular}

the PDR, and reduces the overall network energy consumption, particularly for delay-sensitive applications and also in sparse conditions. The data forwarding protocols without cooperation are focusing on channel conditions that enhance the quality of the received packet at destination; however, transmissions along a single path are influenced as the channel quality varies. The relay selection criteria take into account instantaneous path conditions and distance among neighboring nodes to reliably forward packets to a receiver node in a limited environment of UWSN. Features of multihop and single-hop communication methods have been considered to lower the path-loss and improve the network stability. Optimal weight calculation and act of cooperation provide load balancing in the network and give considerable improvement in the network lifetime.

\section{Conflict of Interests}

The authors declare that there is no conflict of interests regarding the publication of this paper.

\section{References}

[1] A. Umar, M. Akbar, Z. Iqbal, Z. A. Khan, U. Qasim, and N. Javaid, "Cooperative partner nodes selection criteria for cooperative routing in underwater WSNs," in Proceedings of the 5th National Symposium on Information Technology: Towards New Smart World, Wireless Systems and Networks, Dhahran, Saudi Arabia, January 2015.

[2] M. Najimi, A. Ebrahimzadeh, S. M. H. Andargoli, and A. Fallahi, "A novel sensing nodes and decision node selection method for energy efficiency of cooperative spectrum sensing in cognitive sensor networks," IEEE Sensors Journal, vol. 13, no. 5, pp. 1610-1621, 2013.

[3] H. Nasir, N. Javaid, H. Ashraf et al., "CoDBR: cooperative depth based routing for underwater wireless sensor networks," in Proceedings of the 9th IEEE International Conference on Broadband and Wireless Computing, Communication and Applications (BWCCA '14), pp. 52-57, IEEE, Guangdong, China, November 2014.

[4] N. Javaid, M. R. Jafri, Z. A. Khan, U. Qasim, T. A. Alghamdi, and M. Ali, "iAMCTD: improved adaptive mobility of courier nodes in threshold-optimized DBR protocol for underwater wireless sensor networks," International Journal of Distributed Sensor Networks, vol. 2014, Article ID 213012, 12 pages, 2014.

[5] Z. Li, N. Yao, and Q. Gao, "Relative distance based forwarding protocol for underwater wireless networks," International Journal of Distributed Sensor Networks, vol. 2014, Article ID 173089, 11 pages, 2014.

[6] N. Javaid, M. R. Jafri, Z. A. Khan, N. Alrajeh, M. Imran, and A. Vasilakos, "Chain-based communication in cylindrical underwater wireless sensor networks," Sensors, vol. 15, no. 2, pp. 3625-3649, 2015.

[7] X. Wang, M. Xu, H. Wang, Y. Wu, and H. Shi, "Combination of interacting multiple models with the particle filter for threedimensional target tracking in underwater wireless sensor networks," Mathematical Problems in Engineering, vol. 2012, Article ID 829451, 16 pages, 2012.

[8] M. Hammoudeh and R. Newman, "Adaptive routing in wireless sensor networks: QoS optimisation for enhanced application performance," Information Fusion, vol. 22, pp. 3-15, 2015.

[9] H. Wu, X. Chen, C. Shi, Y. Xiao, and M. Xu, "An ACOAAFSA fusion routing algorithm for underwater wireless sensor 
network," International Journal of Distributed Sensor Networks, vol. 2012, Article ID 920505, 9 pages, 2012.

[10] A. Bereketli and S. Bilgen, "Remotely powered underwater acoustic sensor networks," IEEE Sensors Journal, vol. 12, no. 12, pp. 3467-3472, 2012.

[11] Y.-S. Chen and Y.-W. Lin, "Mobicast routing protocol for underwater sensor networks," IEEE Sensors Journal, vol. 13, no. 2, pp. 737-749, 2013.

[12] S. Zhang, D. Li, and J. Chen, "A link-state based adaptive feedback routing for underwater acoustic sensor networks," IEEE Sensors Journal, vol. 13, no. 11, pp. 4402-4412, 2013.

[13] P. K. Chong and D. Kim, "Surface-level path loss modeling for sensor networks in flat and irregular terrain," ACM Transactions on Sensor Networks, vol. 9, no. 2, article 15, 2013.

[14] J. Poncela, M. C. Aguayo, and P. Otero, "Wireless underwater communications," Wireless Personal Communications, vol. 64, no. 3, pp. 547-560, 2012.

[15] G. Han, J. Jiang, N. Bao, L. Wan, and M. Guizani, "Routing protocols for underwater wireless sensor networks," IEEE Communications Magazine, vol. 53, no. 11, pp. 72-78, 2015.

[16] S. Wang, L. Chen, H. Hu, Z. Xue, and W. Pan, "Underwater localization and environment mapping using wireless robots," Wireless Personal Communications, vol. 70, no. 3, pp. 1147-1170, 2013.

[17] C.-M. Chao and M.-W. Lu, "Energy-efficient transmissions for bursty traffic in underwater sensor networks," International Journal of Ad Hoc and Ubiquitous Computing, vol. 13, no. 1, pp. 1-9, 2013.

[18] A. Sánchez, S. Blanc, P. Yuste, A. Perles, and J. J. Serrano, “An ultra-low power and flexible acoustic modem design to develop energy-efficient underwater sensor networks," Sensors, vol. 12, no. 6, pp. 6837-6856, 2012.

[19] Y. Wang, D. Wang, Q. Lu, D. Luo, and W. Fang, "Aquatic debris detection using embedded camera sensors," Sensors, vol. 15, no. 2, pp. 3116-3137, 2015.

[20] Z. Liu, H. Gao, W. Wang, S. Chang, and J. Chen, "Color filtering localization for three dimensional underwater acoustic sensor networks," Sensors, vol. 15, no. 3, pp. 6009-6032, 2015.

[21] M. Xu, G. Liu, and H. Wu, "An energy-efficient routing algorithm for underwater wireless sensor networks inspired by ultrasonic frogs," International Journal of Distributed Sensor Networks, vol. 2014, Article ID 351520, 12 pages, 2014.

[22] J. Cao, J. Dou, and S. Dong, "Balance transmission mechanism in underwater acoustic sensor networks," International Journal of Distributed Sensor Networks, vol. 2015, Article ID 429340, 12 pages, 2015.

[23] H. Chen, Y. Li, J. L. Rebelatto, B. F. Uchoa-Filho, and B. Vucetic, "Harvest-then-cooperate: wireless-powered cooperative communications," IEEE Transactions on Signal Processing, vol. 63, no. 7, pp. 1700-1711, 2015.

[24] S. Wang, H. Liu, and K. Liu, "An improved clustering cooperative spectrum sensing algorithm based on modified doublethreshold energy detection and its optimization in cognitive wireless sensor networks," International Journal of Distributed Sensor Networks, vol. 2015, Article ID 136948, 7 pages, 2015.

[25] M. E. Tipping and C. M. Bishop, "Probabilistic principal component analysis," Journal of the Royal Statistical Society: Series B (Statistical Methodology), vol. 61, no. 3, pp. 611-622, 1999.

[26] R. J. Urick, Principles of Underwater Sound for Engineers, Tata McGraw-Hill Education, 1967.
[27] L. Berkhovskikh and Y. Lysanov, Fundamentals of Ocean Acoustics, Springer, New York, NY, USA, 1982.

[28] W. Zhang and U. Mitra, "A delay-reliability analysis for multihop underwater acoustic communication," in Proceedings of the 2nd Workshop on Underwater Networks (WuWNet'07), pp. 5764, ACM, Montreal, Canada, September 2007.

[29] A. Khandani, J. Abounadi, E. Modiano, and L. Zheng, "Reliability and route diversity in wireless networks," IEEE Transactions on Wireless Communications, vol. 7, no. 12, pp. 4772-4776, 2008. 


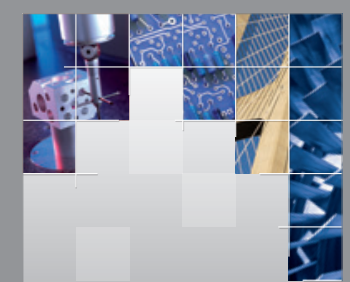

\section{Enfincering}
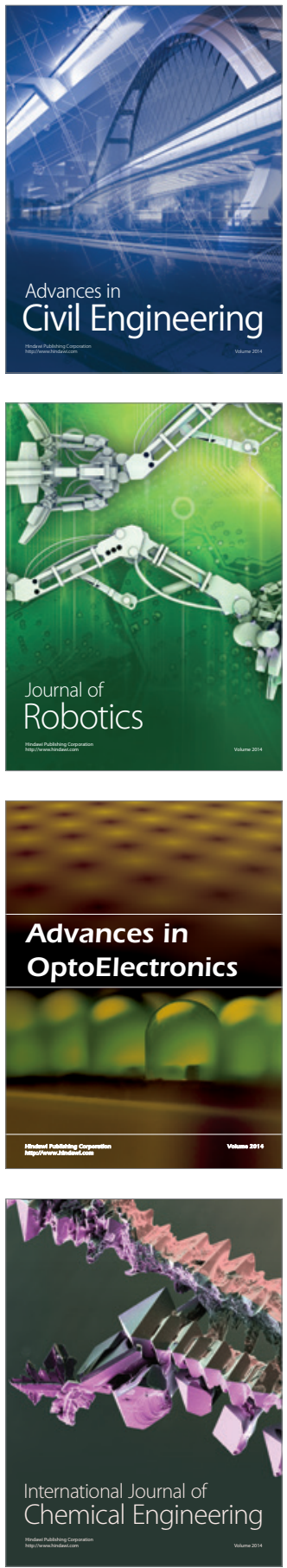

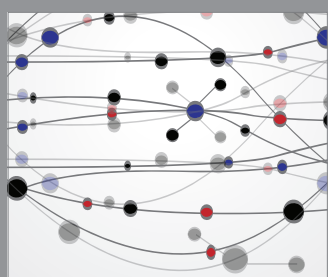

The Scientific World Journal

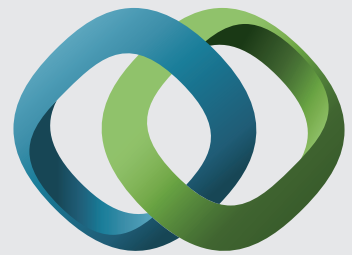

\section{Hindawi}

Submit your manuscripts at

http://www.hindawi.com
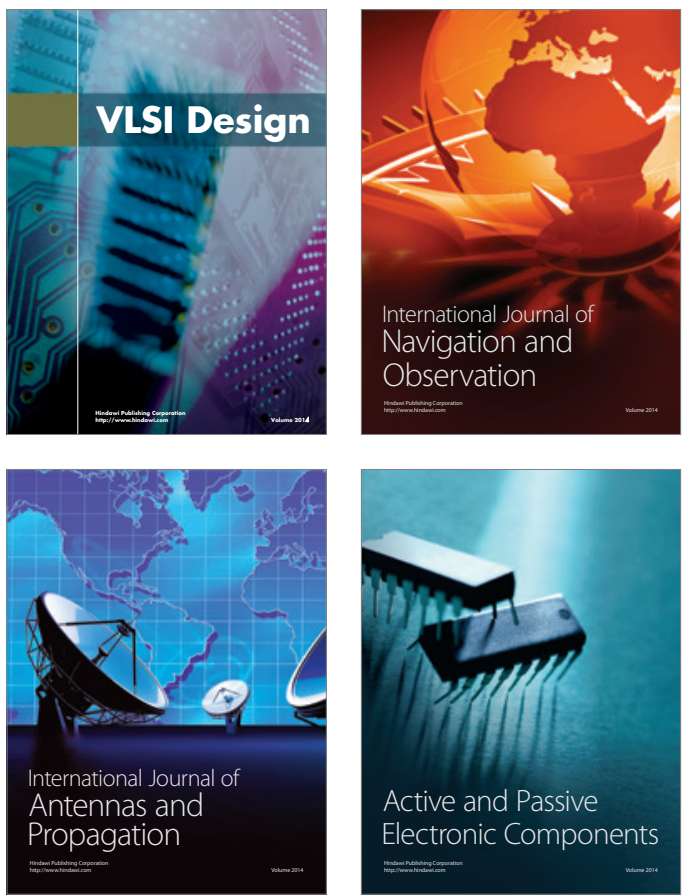
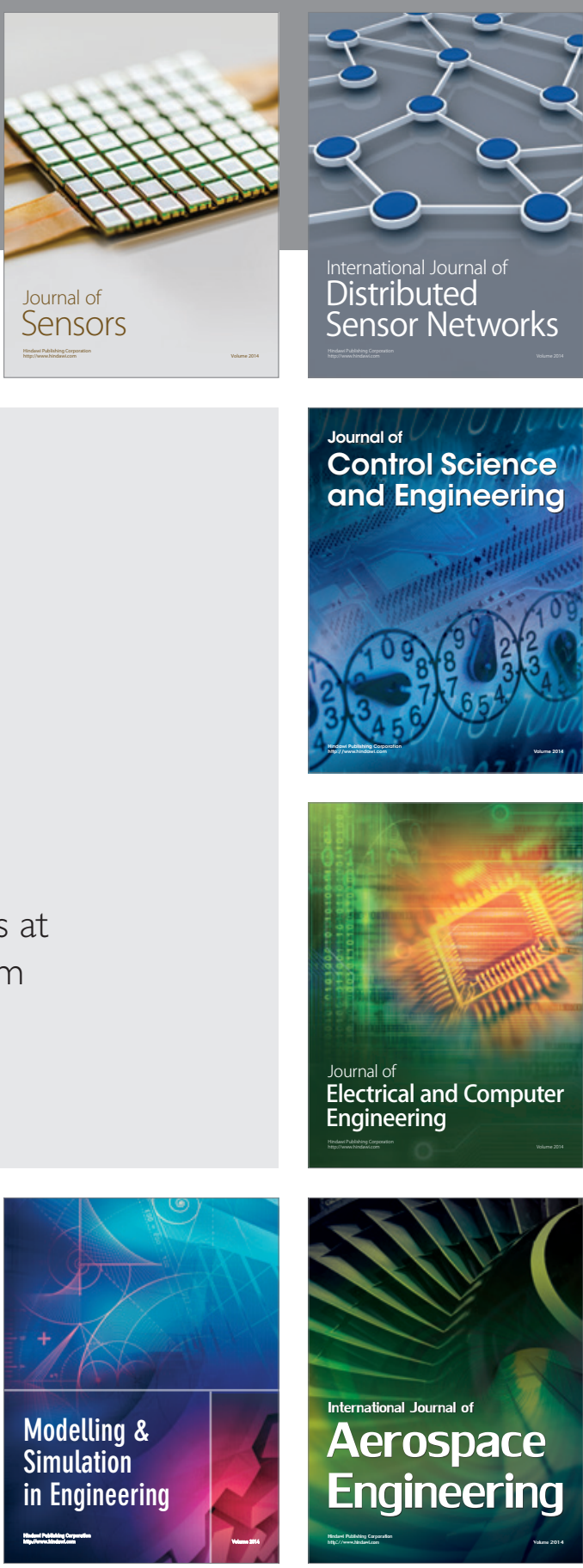

International Journal of

Distributed

Sensor Networks

Journal of

Control Science

and Engineering
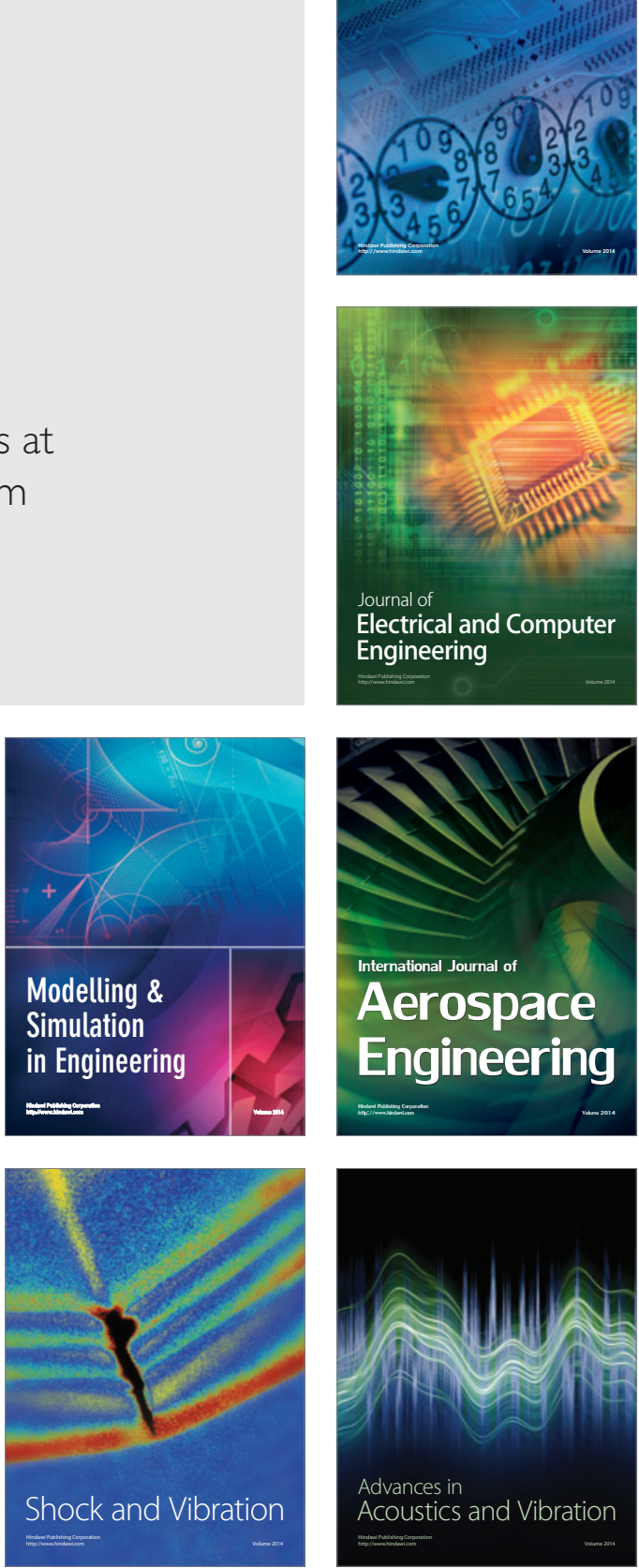